\title{
The Second Main Theorem for Holomorphic Curves into Semi-Abelian Varieties
}

\author{
Junjiro Noguchi, Jörg Winkelmann and Katsutoshi Yamanoi
}

\section{Introduction and main result.}

Let $f: \mathbf{C} \rightarrow A$ be an entire holomorphic curve from the complex plane $\mathbf{C}$ into a semiAbelian variety $A$. It was proved by [No81] that the Zariski closure of $f(\mathbf{C})$ is a translate of a semi-Abelian subvariety of $A$ (logarithmic Bloch-Ochiai's theorem). Let $D$ be an effective algebraic divisor on $A$ which is compactified to $\bar{D}$ on a natural compactification $\bar{A}$ of $A$ (see $\S 3$ ). If $f$ omits $D$, i.e., $f(\mathbf{C}) \cap D=\emptyset$, then $f(\mathbf{C})$ is contained in a translate of a closed subgroup of $A$ that has no intersection with $D$ (see [No98], [SiY96]). Note that the same holds for complex semi-tori defined in $\S 3$ (see [NW99]). In particular, if $A$ is Abelian and $D$ is ample, then $f$ is constant. This was called Lang's conjecture. A similar statement however is found in Bloch [Bl29], p. 55, Théorème K without much proof, and it is not clear what his Théorème $\mathrm{K}$ really means (cf. [Bl29]).

The purpose of the present paper is to establish the quantitative version of the above result for $f$ whose image may intersect $D$, i.e., the second main theorem and the defect relation (cf. $\S \S 2,3$ for the notation):

Main Theorem. Let $f: \mathbf{C} \rightarrow M$ be a holomorphic curve into a complex semi-torus $M$ such that the image $f(\mathbf{C})$ is Zariski dense in $M$. Let $D$ be an effective divisor on $M$ such that the closure $\bar{D}$ of $D$ in $\bar{M}$ is an effective divisor on $\bar{M}$. Assume that $D$ satisfies the boundary condition 1.11 . Then we have the following.

(i) Suppose that $f$ is of finite order $\rho_{f}$. Then there is a positive integer $k_{0}=k_{0}\left(\rho_{f}, D\right)$ depending only on $\rho_{f}$ and $D$ such that

$$
T_{f}\left(r ; c_{1}(\bar{D})\right)=N_{k_{0}}\left(r ; f^{*} D\right)+O(\log r) .
$$

(ii) Suppose that $f$ is of infinite order. Then there is a positive integer $k_{0}=k_{0}(f, D)$ depending on $f$ and $D$ such that

$$
T_{f}\left(r ; c_{1}(\bar{D})\right)=N_{k_{0}}\left(r ; f^{*} D\right)+O\left(\log T_{f}\left(r ; c_{1}(\bar{D})\right)\right)+O(\log r) \|_{E} .
$$


Specially, $\delta(f ; \bar{D})=\delta_{k_{0}}(f ; \bar{D})=0$ in both cases.

See Examples 4.13, 5.18, and Proposition 5.13 that show the necessity of condition 4.11. The most essential part of the proof is the proof of an estimate of the proximity function (see Lemma 5.1):

$$
m_{f}(r ; \bar{D})=O(\log r) \text { or } O\left(\log T_{f}\left(r ; c_{1}(\bar{D})\right)\right)+O(\log r) \|_{E}
$$

Here, when $f$ is of infinite order, we use one idea from R. Kobayashi [Kr98] which is the method of the proof of Proposition (2.14) in [Kr98] (see Lemma 5.4, (ii), and (5.10)). The notion of logarithmic jet spaces due to [No86] plays also a crucial role (cf. [DL97] for an extension to the case of directed jets). We then use the jet projection method developed by [NO $\left.\frac{84}{90}\right]$, Chap. 6, $\S 3$ (cf. [No77], [No81], and [No98]).

In $\S 6$ we will discuss some applications of the Main Theorem.

In [Kr98] R. Kobayashi claimed (1.1) for Abelian A, but there is a part of the arguments which are heuristic, and hard to follow rigorously. Siu-Yeung [SiY97] claimed that for Abelian $A$

$$
m_{f}(r ; D) \leqq \epsilon T_{f}\left(r ; c_{1}(D)\right)+O(\log r) \|_{E(\epsilon)},
$$

where $\epsilon$ is an arbitrarily given positive number, but unfortunately there was a gap in the proof (see Remark 5.30). M. McQuillan [Mc96] dealt with an estimate of type (1.2) for some proper monoidal transformation of $\bar{D} \subset \bar{A}$ with semi-Abelian $A$ by a method different to those mentioned above and ours (see [Mc96], Theorem 1).

It might be appropriate at this point to recall the higher dimensional cases in which the second main theorem has been established. There are actually only a few such cases that have provided fundamental key steps. The first was by H. Cartan [Ca33] for $f: \mathbf{C} \rightarrow$ $\mathbf{P}^{n}(\mathbf{C})$ and hyperplanes in general position, where $\mathbf{P}^{n}(\mathbf{C})$ is the $n$-dimensional complex projective space. The Weyls-Ahlfors theory [Ah41] dealt with the same case and the associated curves as well. W. Stoll [St53/54] generalized the Weyls-Ahlfors theory to the case of $f: \mathbf{C}^{m} \rightarrow \mathbf{P}^{n}(\mathbf{C})$. Griffiths et al., [CG72], [GK73], established the second main theorem for $f: W \rightarrow V$ with a complex affine algebraic variety $W$ and a general complex projective manifold $V$ such that $\operatorname{rank} d f=\operatorname{dim} V$, which was developed well by many others. For $f: \mathbf{C} \rightarrow V$ in general, only an inequality of the second main theorem type such as (5.29) was proved ([No77 96], [AN91]). Eremenko and Sodin [ES92] proved a weak second main theorem for $f: \mathbf{C} \rightarrow \mathbf{P}^{n}(\mathbf{C})$ and hypersurfaces in general position, where the counting functions are not truncated. In this sense, the Main Theorem adds a new case in which an explicit second main theorem is established.

Acknowledgement. The authors are grateful to Professor Ryoichi Kobayashi for interesting discussions and his idea mentioned above. 


\section{Order functions.}

(a) For a general reference of items presented in this section, cf., e.g., $\left[\mathrm{NO} \frac{84}{90}\right]$. First we recall some standard notation. Let $\phi$ and $\psi$ be functions in a variable $r>0$ such that $\psi>0$. Let $E$ be a measurable subset of real positive numbers with finite measure. Then the expression

$$
\phi(r)=O(\psi(r)) \quad\left(\operatorname{resp} . \phi(r)=O(\psi(r)) \|_{E}\right)
$$

stands for

$$
\left.\varlimsup_{r \rightarrow \infty} \frac{|\phi(r)|}{\psi(r)}<\infty \quad \text { (resp. } \varlimsup_{r \rightarrow \infty, r \notin E} \frac{|\phi(r)|}{\psi(r)}<\infty\right) .
$$

Specially, $O(1)$ denotes a bounded term.

We use the superscript ${ }^{+}$to denote the positive part, e.g., $\log ^{+} r=\max \{0, \log r\}$. We write $\mathbf{R}^{+}$for the set of all real positive numbers. We denote by $\Re z$ (resp. $\Im z$ ) the real (resp. imaginary) part of a complex number $z \in \mathbf{C}$.

(b) Let $X$ be a compact Kähler manifold and let $\omega$ be a real $(1,1)$-form on $X$. For an entire holomorphic curve $f: \mathbf{C} \rightarrow X$ we first define the order function of $f$ with respect to $\omega$ by

$$
T_{f}(r ; \omega)=\int_{0}^{r} \frac{d t}{t} \int_{\Delta(t)} f^{*} \omega,
$$

where $\Delta(t)=\{z \in \mathbf{C} ;|z|<t\}$ is the disk of radius $t$ with center at the origin of the complex plane $\mathbf{C}$. Let $[\omega] \in H^{2}(X, \mathbf{R})$ be a second cohomology class represented by a closed real $(1,1)$-form $\omega$ on $X$. Then we set

$$
T_{f}(r ;[\omega])=T_{f}(r ; \omega)
$$

Let $\left[\omega^{\prime}\right]=[\omega]$ be another representation of the class. Since $X$ is compact Kähler, there is a smooth function $b$ on $X$ such that $(i / 2 \pi) \partial \bar{\partial} b=\omega^{\prime}-\omega$. There is a positive constant $C$ with $|b| \leqq C$. Then by Jensen's formula (cf. [NO $\left.\frac{84}{90}\right]$, Lemma (3.39) and Remark (5.2.21)) we have

$$
\left|T_{f}\left(r ; \omega^{\prime}\right)-T_{f}(r ; \omega)\right| \leqq C .
$$

Therefore, the order function $\left.T_{f}(r ; \omega]\right)$ of $f$ with respect to the cohomology class $[\omega]$ is well-defined up to a bounded term. Taking a positive definite form $\omega$ on $X$, we define the order of $f$ by

$$
\rho_{f}=\varlimsup_{r \rightarrow \infty} \frac{\log T_{f}(r ; \omega)}{\log r} \leqq \infty,
$$

which is independent of the choice of such $\omega$. We say that $f$ is of finite order if $\rho_{f}<\infty$.

Let $D$ be an effective divisor on $X$. We denote by $\operatorname{Supp} D$ the support of $D$, but sometimes write simply $D$ for $\operatorname{Supp} D$ if there is no confusion. Assume that $f(\mathbf{C}) \not \subset D$. Let $L(D)$ be the line bundle determined by $D$ and let $\sigma \in H^{0}(X, L(D))$ be a global 
holomorphic section of $L(D)$ whose divisor $(\sigma)$ is $D$. Take a hermitian fiber metric $\|\cdot\|$ in $L(D)$ with curvature form $\omega$, normalized so that $\omega$ represents the first Chern class $c_{1}(L(D))$ of $L(D) ; c_{1}(L(D))$ will be abbreviated to $c_{1}(D)$. Set

$$
\begin{aligned}
T_{f}\left(r ; c_{1}(D)\right) & =T_{f}(r ; \omega), \\
m_{f}(r ; D) & =\frac{1}{2 \pi} \int_{0}^{2 \pi} \log \frac{1}{\left\|\sigma\left(f\left(r e^{i \theta}\right)\right)\right\|} d \theta .
\end{aligned}
$$

It is known that if $D$ is ample, then $f$ is rational if and only if

$$
\varliminf_{r \rightarrow \infty} \frac{T_{f}\left(r ; c_{1}(\bar{D})\right)}{\log r}<\infty .
$$

One sometimes writes $T_{f}(r ; L(D))$ for $T_{f}\left(r ; c_{1}(D)\right)$, but it is noted that $T_{f}\left(r ; c_{1}(D)\right)$ is not depending on a specific choice of $D$ in the homology class. We call $m_{f}(r ; D)$ the proximity function of $f$ for $D$. Denoting by $\operatorname{ord}_{z} f^{*} D$ the order of the pull-backed divisor $f^{*} D$ at $z \in \mathbf{C}$, we set

$$
\begin{aligned}
n\left(t ; f^{*} D\right) & =\sum_{z \in \Delta(t)} \operatorname{ord}_{z} f^{*} D, \\
n_{k}\left(t ; f^{*} D\right) & =\sum_{z \in \Delta(t)} \min \left\{k, \operatorname{ord}_{z} f^{*} D\right\} \\
N\left(r ; f^{*} D\right) & =\int_{1}^{r} \frac{n\left(t ; f^{*} D\right)}{t} d t \\
N_{k}\left(r ; f^{*} D\right) & =\int_{1}^{r} \frac{n_{k}\left(t ; f^{*} D\right)}{t} d t
\end{aligned}
$$

These are called the counting functions of $f^{*} D$. Then we have the F.M.T. (First Main Theorem) (cf. [NO $\left.\frac{84}{90}\right]$, Chap. Chap. V):

$$
T_{f}\left(r ; c_{1}(D)\right)=N\left(r ; f^{*} D\right)+m_{f}(r ; D)+O(1) .
$$

The quantities

$$
\begin{gathered}
\delta(f ; D)=1-\varlimsup_{r \rightarrow \infty} \frac{N\left(r ; f^{*} D\right)}{T_{f}(r ; L(D))} \in[0,1], \\
\delta_{k}(f ; D)=1-\varlimsup_{r \rightarrow \infty} \frac{N_{k}\left(r ; f^{*} D\right)}{T_{f}(r ; L(D))} \in[0,1]
\end{gathered}
$$

are called the defects of $f$ for $D$.

(c) Let $F(z)$ be a meromorphic function, and let $(F)_{\infty}\left(\operatorname{resp} .(F)_{0}\right)$ denote the polar (resp. zero) divisor of $F$. Define the proximity function of $F(z)$ by

$$
m(r, F)=\frac{1}{2 \pi} \int_{0}^{2 \pi} \log ^{+}\left|F\left(r e^{i \theta}\right)\right| d \theta .
$$


Nevanlinna's order function is defined by

$$
T(r, F)=m(r, F)+N\left(r ;(F)_{\infty}\right) .
$$

Cf., e.g., $\left[\mathrm{NO} \frac{84}{90}\right]$, Chap. 6 for the basic properties of $T(r, F)$. For instance, let $T_{F}(r ; \omega)$ be the order function of holomorphic $F: \mathbf{C} \rightarrow \mathbf{P}^{1}(\mathbf{C})$ with respect to the Fubini-Study metric form $\omega$. Then Shimizu-Ahlfors' theorem says that

$$
T_{F}(r ; \omega)-T(r, F)=O(1) .
$$

If $F \not \equiv 0, T(r, 1 / F)=m(r, 1 / F)+N\left(r ;(F)_{0}\right)$, and then by Nevanlinna's F.M.T. (cf. $\left.[\mathrm{Ha} 64],\left[\mathrm{NO} \frac{84}{90}\right]\right)$

$$
T(r, F)=T\left(r, \frac{1}{F}\right)+O(1) .
$$

For several meromorphic functions $F_{j}, 1 \leqq j \leqq l$, on $\mathbf{C}$ we have

$$
\begin{aligned}
T\left(r, \prod_{j=1}^{l} F_{j}\right) & \leqq \sum_{j=1}^{l} T\left(r, F_{j}\right), \\
T\left(r, \sum_{j=1}^{l} F_{j}\right) & \leqq \sum_{j=1}^{l} T\left(r, F_{j}\right)+\log l, \\
T\left(r, R\left(F_{1}, \ldots, F_{l}\right)\right) & \leqq O\left(\sum_{j=1}^{l} T\left(r, F_{j}\right)\right)+O(1),
\end{aligned}
$$

where $R\left(F_{1}, \ldots, F_{l}\right)$ is a rational function in $F_{1}, \ldots, F_{l}$ and $R\left(F_{1}(z), \ldots, F_{l}(z)\right) \not \equiv \infty$.

Lemma 2.4 (cf., [NO $\left.\frac{84}{90}\right]$, Theorem (5.2.29)) Let $X$ be a compact Kähler manifold, let $L$ be a hermitian line bundle on $X$, and let $\sigma_{1}, \sigma_{2} \in H^{0}(X, L)$ with $\sigma_{1} \not \equiv 0$. Let $f: \mathbf{C} \rightarrow X$ be a holomorphic curve such that $f(\mathbf{C}) \not \subset \operatorname{Supp}\left(\sigma_{1}\right)$. Then we have

$$
T\left(r, \frac{\sigma_{2}}{\sigma_{1}} \circ f\right) \leqq T_{f}\left(r ; c_{1}(L)\right)+O(1) .
$$

Proof. It follows from the definition that

$$
N\left(r ;\left(\frac{\sigma_{2}}{\sigma_{1}} \circ f\right)_{\infty}\right) \leqq N\left(r ; f^{*}\left(\sigma_{1}\right)\right) .
$$

Moreover, we have

$$
\begin{aligned}
m\left(r, \frac{\sigma_{2}}{\sigma_{1}} \circ f\right) & =\frac{1}{2 \pi} \int_{\{|z|=r\}} \log ^{+} \frac{\left\|\sigma_{2} \circ f\right\|}{\left\|\sigma_{1} \circ f\right\|} d \theta \\
& \leqq \frac{1}{2 \pi} \int_{\{|z|=r\}} \log ^{+} \frac{1}{\left\|\sigma_{1} \circ f\right\|} d \theta+O(1) .
\end{aligned}
$$


Thus the required estimate follows from these and (2.1). Q.E.D.

(d) We begin with introducing a notation for a small term.

Definition. We write $S_{f}\left(r ; c_{1}(D)\right)$, sometimes $S_{f}(r ; L(D))$, to express a small term such that

$$
S_{f}\left(r ; c_{1}(D)\right)=O(\log r)
$$

if $T_{f}\left(r ; c_{1}(D)\right)$ is of finite order, and

$$
S_{f}\left(r ; c_{1}(D)\right)=O\left(\log T_{f}\left(r ; c_{1}(D)\right)\right)+O(\log r) \|_{E},
$$

otherwise. We use the notation $S_{f}(r ; \omega)$ in the same sense as above with respect to $T_{f}(r ; \omega)$. For a meromorphic function $F$ on $\mathbf{C}$, the notation $S(r, F)$ is used to express a small term with respect to $T(r, F)$ as well.

Lemma 2.5 (i) Let $F$ be a meromorphic function and let $F^{(k)}(z)$ be the $k$-th derivative of $F$ for $k=1,2, \ldots$ Then

$$
m\left(r, \frac{F^{(k)}}{F}\right)=S(r, F) .
$$

Moreover, if $F$ is entire,

$$
T\left(r, F^{(k)}\right)=T(r, F)+S(r, F), \quad k \geqq 1 .
$$

(ii) Let the notation be as in Lemma 2.4, and set $\varphi(z)=\frac{\sigma_{2}}{\sigma_{1}} \circ f(z)$. Suppose that $\varphi \not \equiv 0$. Then

$$
m\left(r, \frac{\varphi^{(k)}}{\varphi}\right)=S_{f}\left(r, c_{1}(D)\right), \quad k \geqq 1 \text {. }
$$

Proof. The item (i) is called Nevanlinna's lemma on logarithmic derivatives (cf. [NO $\left.\frac{84}{90}\right]$, Corollary (6.1.19)). Then (ii) follows from (i) and Lemma 2.4. Q.E.D.

The following is called Borel's lemma (cf. [Ha64], p. 38, Lemma 2.4).

Lemma 2.6 Let $\phi(r)$ be a continuous, increasing function on $\mathbf{R}^{+}$such that $\phi\left(r_{0}\right)>0$ for some $r_{0} \in \mathbf{R}^{+}$. Then we have

$$
\phi\left(r+\frac{1}{\phi(r)}\right)<2 \phi(r) \|_{E} .
$$

For a later use we show

Lemma 2.7 Let $F$ be an entire function, and let $0<r<R$.

(i) $T(r, F)=m(r, F) \leqq \max _{|z|=r} \log |F(z)| \leqq \frac{R+r}{R-r} m(R, F)$. 
(ii) $m(r, F)=S\left(r, e^{2 \pi i F}\right)$.

Proof. (i) See $\left[\mathrm{NO} \frac{84}{90}\right]$, Theorem (5.3.13) or [Ha64], p. 18, Theorem 1.6.

(ii) Using the complex Poisson kernel, we have

$$
F(z)=\frac{i}{2 \pi} \int_{\{|\zeta|=R\}} \frac{\zeta+z}{\zeta-z} \Im F(\zeta) d \theta+\Re F(0) .
$$

Therefore, using (i) and the F.M.T. (2.2) with $0<r<R<R^{\prime}$ we obtain

$$
\begin{aligned}
\max _{|z|=r}|F(z)| & \leqq \frac{R+r}{R-r} \max _{|\zeta|=R}|\Im F(\zeta)|+|\Re F(0)| \\
& \leqq \frac{R+r}{R-r}\left(\max _{|\zeta|=R} \Im F(\zeta)+\max _{|\zeta|=R} \Im(-F(\zeta))\right)+|\Re F(0)| \\
& \leqq \frac{R+r}{R-r} \cdot \frac{R^{\prime}+R}{R^{\prime}-R} \cdot \frac{1}{2 \pi}\left(m\left(R^{\prime}, e^{-2 \pi i F}\right)+m\left(R^{\prime}, e^{2 \pi i F}\right)\right)+|\Re F(0)| \\
& \leqq \frac{R+r}{R-r} \cdot \frac{R^{\prime}+R}{R^{\prime}-R} \cdot \frac{1}{\pi}\left(T\left(R^{\prime}, e^{2 \pi i F}\right)+O(1)\right)+O(1) .
\end{aligned}
$$

If $T\left(r, e^{2 \pi i F}\right)$ has finite order, then setting $R=2 r$ and $R^{\prime}=3 r$, we see by (2.8) that

$$
m(r, F) \leqq \log \max _{|z|=r}|F(z)|=O(\log r) .
$$

In the case where $T\left(r, e^{2 \pi i F}\right)$ has infinite order, we write $T(r)=T\left(r, e^{2 \pi i F}\right)$ for the sake of simplicity. Setting $R=r+\frac{1}{2 T(r)}$ and $R^{\prime}=r+\frac{1}{T(r)}$, we have by (2.8) and Lemma 2.6

$$
\begin{aligned}
m(r, F) & \leqq \log \max _{|z|=r}|F(z)| \\
& \leqq \log \left((4 r T(r)+1)(4 r T(r)+3)\left(T\left(r+\frac{1}{T(r)}\right)+O(1)\right)+O(1)\right) \\
& \leqq \log ((4 r T(r)+1)(4 r T(r)+3)(2 T(r)+O(1))+O(1)) \|_{E} \\
& =S\left(r, e^{2 \pi i F}\right) .
\end{aligned}
$$

Q.E.D.

\section{Complex semi-torus.}

Let $M$ be a complex Lie group admitting the exact sequence

$$
0 \rightarrow\left(\mathbf{C}^{*}\right)^{p} \rightarrow M \stackrel{\eta}{\rightarrow} M_{0} \rightarrow 0
$$

where $\mathbf{C}^{*}$ is the multiplicative group of non-zero complex numbers, and $M_{0}$ is a (compact) complex torus. Such $M$ is called a complex semi-torus or a quasi-torus. If $M_{0}$ is algebraic, 
that is, an Abelian variety, $M$ is called a semi-Abelian variety or a quasi-Abelian variety. In this section and in the next, we assume that $M$ is a complex semi-torus.

Taking the universal coverings of (3.1), one gets

$$
0 \rightarrow \mathbf{C}^{p} \rightarrow \mathbf{C}^{n} \rightarrow \mathbf{C}^{m} \rightarrow 0
$$

and an additive discrete subgroup $\Lambda$ of $\mathbf{C}^{n}$ such that

$$
\begin{aligned}
\pi: \mathbf{C}^{n} & \rightarrow M=\mathbf{C}^{n} / \Lambda, \\
\pi_{0}: \mathbf{C}^{m}=\left(\mathbf{C}^{n} / \mathbf{C}^{p}\right) & \rightarrow M_{0}=\left(\mathbf{C}^{n} / \mathbf{C}^{p}\right) /\left(\Lambda / \mathbf{C}^{p}\right), \\
\left(\mathbf{C}^{*}\right)^{p} & =\mathbf{C}^{p} /\left(\Lambda \cap \mathbf{C}^{p}\right) .
\end{aligned}
$$

We fix a linear complex coordinate system $x=\left(x^{\prime}, x^{\prime \prime}\right)=\left(x_{1}^{\prime}, \ldots, x_{p}^{\prime}, x_{1}^{\prime \prime}, \ldots, x_{m}^{\prime \prime}\right)$ on $\mathbf{C}^{n}$ such that $\mathbf{C}^{p} \cong\left\{x_{1}^{\prime \prime}=\cdots=x_{m}^{\prime \prime}=0\right\}$ and

$$
\Lambda \cap \mathbf{C}^{p}=\mathbf{Z}\left(\begin{array}{c}
1 \\
\vdots \\
0
\end{array}\right)+\cdots+\mathbf{Z}\left(\begin{array}{c}
0 \\
\vdots \\
1
\end{array}\right) .
$$

The covering mapping $\mathbf{C}^{p} \rightarrow\left(\mathbf{C}^{*}\right)^{p}$ is given by

$$
\left(\begin{array}{c}
x_{1} \\
\vdots \\
x_{p}
\end{array}\right) \in \mathbf{C}^{p} \rightarrow\left(\begin{array}{c}
e^{2 \pi i x_{1}} \\
\vdots \\
e^{2 \pi i x_{p}}
\end{array}\right)=\left(\begin{array}{c}
u_{1} \\
\vdots \\
u_{p}
\end{array}\right) \in\left(\mathbf{C}^{*}\right)^{p} .
$$

We may regard $\eta: M \rightarrow M_{0}$ to be a flat $\left(\mathbf{C}^{*}\right)^{p}$-principal fiber bundle. By a suitable change of coordinates $\left(x_{1}^{\prime}, \ldots, x_{p}^{\prime}, x_{1}^{\prime \prime}, \ldots, x_{m}^{\prime \prime}\right)$ the discrete group $\Lambda$ is generated over $\mathbf{Z}$ by the column vectors of the matrix of the following type

$$
\left(\begin{array}{cccc}
1 & \cdots & 0 & \\
\vdots & \ddots & \vdots & A \\
0 & \cdots & 1 & \\
& O & & B
\end{array}\right)
$$

where $A$ is a real $(p, m)$-matrix and $C$ is a $(m, m)$-matrix. Therefore the transition matrixfunctions of the flat $\left(\mathbf{C}^{*}\right)^{p}$-principal fiber bundle $\eta: M \rightarrow M_{0}$ are expressed by a diagonal matrix such that

$$
\left(\begin{array}{cccc}
a_{1} & 0 & \cdots & 0 \\
0 & a_{2} & \cdots & 0 \\
\vdots & \vdots & \ddots & \vdots \\
0 & 0 & \cdots & a_{p}
\end{array}\right), \quad\left|a_{1}\right|=\cdots=\left|a_{p}\right|=1
$$


Taking the natural compactification $\mathbf{C}^{*}=\mathbf{P}^{1}(\mathbf{C}) \backslash\{0, \infty\} \hookrightarrow \mathbf{P}^{1}(\mathbf{C})$, we have a compactification of $M$,

$$
\bar{\eta}: \bar{M} \rightarrow M_{0}
$$

which is a flat $\left(\mathbf{P}^{1}(\mathbf{C})\right)^{p}$-fiber bundle over $M_{0}$. Set

$$
\partial M=\bar{M} \backslash M
$$

which is a divisor on $\bar{M}$ with only simple normal crossings.

Let $\Omega_{1}$ be the product of the Fubini-Study metric forms on $\left(\mathbf{P}^{1}(\mathbf{C})\right)^{p}$,

$$
\Omega_{1}=\frac{i}{2 \pi} \sum_{j=1}^{p} \frac{d u_{j} \wedge d \bar{u}_{j}}{\left(1+\left|u_{j}\right|^{2}\right)^{2}} .
$$

Because of (3.3) $\Omega_{1}$ is defined well on $\bar{M}$. Let $\Omega_{2}=(i / 2 \pi) \partial \bar{\partial} \sum_{j}\left|x_{j}^{\prime \prime}\right|^{2}$ be the flat hermitian metric form on $\mathbf{C}^{m}$, and as well on the complex torus $M_{0}$. Then we set

$$
\Omega=\Omega_{1}+\bar{\eta}^{*} \Omega_{2}
$$

which is a Kähler form on $\bar{M}$.

Remark. The same complex Lie group $M$ may admit several such exact sequences as (3.1) which may be quite different. For instance, let $\tau$ be an arbitrary complex number with $\Im \tau>0$. Let $\Lambda$ be the discrete subgroup of $\mathbf{C}^{2}$ generated by

$$
\left(\begin{array}{l}
1 \\
0
\end{array}\right),\left(\begin{array}{l}
0 \\
1
\end{array}\right),\left(\begin{array}{l}
i \\
\tau
\end{array}\right) .
$$

Then $M=\mathbf{C}^{2} / \Lambda$ is a complex semi-torus and the natural projection of $\mathbf{C}^{2}=\mathbf{C} \times \mathbf{C}$ onto the first and the second factors induce respectively exact sequences of the forms

$$
\begin{aligned}
& 0 \rightarrow \mathbf{C}^{*} \rightarrow M \rightarrow \mathbf{C} /\langle 1, i\rangle_{\mathbf{Z}} \rightarrow 0 \\
& 0 \rightarrow \mathbf{C}^{*} \rightarrow M \rightarrow \mathbf{C} /\langle 1, \tau\rangle_{\mathbf{Z}} \rightarrow 0 .
\end{aligned}
$$

In the sequel we always consider a complex semi-torus $M$ with a fixed exact sequence as in (3.1) and with the discrete subgroup $\Lambda$ satisfying (3.2).

Let $f: \mathbf{C} \rightarrow M$ be a holomorphic curve. We regard $f$ as a holomorphic curve into $\bar{M}$ equipped with the Kähler form $\Omega$, and define the order function by

$$
T_{f}(r ; \Omega)=\int_{0}^{r} \frac{d t}{t} \int_{\Delta(t)} f^{*} \Omega .
$$

Let $\tilde{f}: \mathbf{C} \rightarrow \mathbf{C}^{n}$ be the lifting of $f$, and set

$$
\tilde{f}(z)=\left(F_{1}(z), \ldots, F_{p}(z), G_{1}(z), \ldots, G_{m}(z)\right)
$$


where $F_{i}(z)$ and $G_{j}(z)$ are entire functions. Extending the base $M_{0}$ of the fiber bundle $M \rightarrow M_{0}$ to the universal covering $\pi_{0}: \mathbf{C}^{m} \rightarrow M_{0}$, we have

$$
M \times{ }_{M_{0}} \mathbf{C}^{m} \cong\left(\mathbf{C}^{*}\right)^{p} \times \mathbf{C}^{m}, \quad \bar{M} \times{ }_{M_{0}} \mathbf{C}^{m} \cong\left(\mathbf{P}^{1}(\mathbf{C})\right)^{p} \times \mathbf{C}^{m}
$$

Set

$$
\hat{M}=\left(\mathbf{P}^{1}(\mathbf{C})\right)^{p} \times \mathbf{C}^{m} .
$$

Then $\hat{M}$ is the universal covering of $\bar{M}$, and then $\tilde{f}$ induces a lifting $\hat{f}$ of $f: \mathbf{C} \rightarrow M \hookrightarrow \bar{M}$,

$$
\hat{f}: z \in \mathbf{C} \rightarrow\left(e^{2 \pi i F_{1}(z)}, \ldots, e^{2 \pi i F_{p}(z)}, G_{1}(z), \ldots, G_{m}(z)\right) \in\left(\mathbf{C}^{*}\right)^{p} \times \mathbf{C}^{m}=\hat{M} .
$$

Set

$$
\begin{aligned}
& \hat{f}_{(1)}: z \in \mathbf{C} \rightarrow\left(e^{2 \pi i F_{1}(z)}, \ldots, e^{2 \pi i F_{p}(z)}\right) \in\left(\mathbf{C}^{*}\right)^{p} \\
& \hat{f}_{(2)}: z \in \mathbf{C} \rightarrow\left(G_{1}(z), \ldots, G_{m}(z)\right) \in \mathbf{C}^{m}
\end{aligned}
$$

By definition we have

$$
T_{f}(r ; \Omega)=T_{\hat{f}_{(1)}}\left(r ; \Omega_{1}\right)+T_{\hat{f}_{(2)}}\left(r ; \Omega_{2}\right) .
$$

By Shimizu-Ahlfors' theorem we have

$$
T_{\hat{f}_{(1)}}\left(r ; \Omega_{1}\right)=\sum_{j=1}^{p} T\left(r, e^{2 \pi i F_{j}}\right)+O(1) .
$$

By Jensen's formula (cf. [NO $\left.\frac{84}{90}\right]$, Lemma (3.3.17)) we have

$$
\begin{aligned}
T_{\hat{f}_{(2)}}\left(r ; \Omega_{2}\right) & =\int_{0}^{r} \frac{d t}{t} \int_{\Delta(t)} \frac{i}{2 \pi} \partial \bar{\partial} \sum_{j=1}^{m}\left|G_{j}(z)\right|^{2} \\
& =\frac{1}{4 \pi} \int_{0}^{2 \pi}\left(\sum_{j=1}^{m}\left|G_{j}\left(r e^{i \theta}\right)\right|^{2}\right) d \theta-\frac{1}{2} \sum_{j=1}^{m}\left|G_{j}(0)\right|^{2} .
\end{aligned}
$$

Lemma 3.8 Let the notation be as above. Then for $k \geqq 0$ we have

$$
\begin{aligned}
& T\left(r, F_{j}^{(k)}\right)=T\left(r, F_{j}\right)+k S\left(r, F_{j}\right) \leqq S_{\hat{f}_{(1)}}\left(r ; \Omega_{1}\right) \leqq S_{f}(r ; \Omega), \\
& T\left(r, G_{j}^{(k)}\right)=T\left(r, G_{j}\right)+k S\left(r, G_{j}\right) \leqq S_{\hat{f}_{(2)}}\left(r ; \Omega_{2}\right) \leqq S_{f}(r ; \Omega) .
\end{aligned}
$$


Proof. By Lemma 2.5 it suffices to show the case of $k=0$. By Lemma 2.7 and (3.6)

$$
T\left(r, F_{j}\right)=m\left(r, F_{j}\right)=S\left(r, e^{2 \pi i F_{j}}\right) \leqq S_{\hat{f}_{(1)}}\left(r ; \Omega_{1}\right) \leqq S_{f}(r ; \Omega) .
$$

For $G_{j}$ we have by making use of (3.7) and the concavity of the logarithmic function

$$
\begin{aligned}
T\left(r ; G_{j}\right) & =m\left(r, G_{j}\right)=\frac{1}{2 \pi} \int_{\{|z|=r\}} \log ^{+}\left|G_{j}(z)\right| d \theta \\
& =\frac{1}{4 \pi} \int_{\{|z|=r\}} \log ^{+}\left|G_{j}(z)\right|^{2} d \theta \\
& \leqq \frac{1}{4 \pi} \int_{\{|z|=r\}} \log \left(1+\left|G_{j}(z)\right|^{2}\right) d \theta \\
& \leqq \frac{1}{2} \log \left(1+\frac{1}{2 \pi} \int_{\{|z|=r\}}\left|G_{j}(z)\right|^{2} d \theta\right) \\
& =S_{\hat{f}_{(2)}}\left(r ; \Omega_{2}\right) \leqq S_{f}(r ; \Omega) .
\end{aligned}
$$

Q.E.D.

Lemma 3.9 Let the notation be as above. Assume that $f: \mathbf{C} \rightarrow M$ has a finite order $\rho_{f}$. Then $F_{j}(z), 1 \leqq j \leqq p$, are polynomials of degree at most $\rho_{f}$, and $G_{k}, 1 \leqq k \leqq m$, are polynomials of degree at most $\rho_{f} / 2$; moreover, at least one of $F_{j}$ has degree $\rho_{f}$, or at least one of $G_{k}$ has degree $\rho_{f} / 2$.

Proof. Let $\epsilon>0$ be an arbitrary positive number. Then there is a $r_{0}>0$ such that

$$
T_{f}(r ; \Omega) \leqq r^{\rho_{f}+\epsilon}, \quad r \geqq r_{0} .
$$

It follows from $(3.5) \sim(3.7)$ that for $r \geqq r_{0}$

$$
\begin{aligned}
T_{\hat{f}_{(1)}}\left(r ; \Omega_{1}\right) & \leqq r^{\rho_{f}+\epsilon} \\
\frac{1}{2 \pi} \int_{0}^{2 \pi}\left(\sum_{j=1}^{m}\left|G_{j}\left(r e^{i \theta}\right)\right|^{2}\right) d \theta & \leqq r^{\rho_{f}+\epsilon}
\end{aligned}
$$

It follows from (3.10), (3.6), and (2.8) applied with $R=2 r$ and $R^{\prime}=3 r$ that there is a positive constant $C$ such that

$$
\max _{|z|=r}|F(z)| \leqq C r^{\rho_{f}+\epsilon} .
$$

Therefore, $F_{j}(z)$ is a polynomial of degree at most $\rho_{f}$.

Expand $G_{j}(z)=\sum_{\nu}^{\infty} c_{j \nu} z^{\nu}$. Then one gets

$$
\frac{1}{2 \pi} \int_{0}^{2 \pi}\left(\sum_{j=1}^{m}\left|G_{j}\left(r e^{i \theta}\right)\right|^{2}\right) d \theta=\sum_{j=1}^{m} \sum_{\nu=0}^{\infty}\left|c_{j \nu}\right|^{2} r^{2 \nu}
$$


It follows that

$$
\sum_{j=1}^{m} \sum_{\nu=1}^{\infty}\left|c_{j \nu}\right|^{2} r^{2 \nu} \leqq r^{\rho_{f}+\epsilon}, \quad r \geqq r_{0} .
$$

Hence, $c_{j \nu}=0$ for all $\nu>\rho_{f} / 2$ and $1 \leqq j \leqq m$. We see that $G_{j}(z), 1 \leqq j \leqq m$, are polynomials of degree at most $\rho_{f} / 2$. The remaining part is clear. Q.E.D.

In the language of Lie group theory we obtain the following characterization of holomorphic curves of finite order:

Proposition 3.11 Let $M$ be an n-dimensional complex semi-torus with the above compactification $\bar{M}$, let $\operatorname{Lie}(M)$ be its Lie algebra, and let $\exp : \operatorname{Lie}(M) \rightarrow M$ be the exponential map. Let $f: \mathbf{C} \rightarrow M$ be a holomorphic curve. Then $f$ is of finite order considered as a holomorphic curve into $\bar{M}$ if and only if there is a polynomial map $P: \mathbf{C} \rightarrow \operatorname{Lie}(M) \cong \mathbf{C}^{n}$ such that $f=\exp \circ P$, and hence the property of $f$ being of finite order is independent of the choice of the compactification $\bar{M} \supset M$.

\section{Divisors on semi-tori.}

(a) Let $M$ be a complex semi-torus as before:

$$
\begin{aligned}
0 \rightarrow\left(\mathbf{C}^{*}\right)^{p} & \rightarrow M \stackrel{\eta}{\rightarrow} M_{0} \rightarrow 0 \\
\bar{\eta} & : \bar{M} \rightarrow M_{0} .
\end{aligned}
$$

Let $D$ be an effective divisor on $M$ such that $D$ is compactified to $\bar{D}$ in $\bar{M}$; that is, roughly speaking, $D$ is algebraic along the fibers of $M \rightarrow M_{0}$. If $M$ is a semi-Abelian variety, then this condition is equivalent to the algebraicity of $D$. We equip $L(\bar{D}) \rightarrow \bar{M}$ with a hermitian fiber metric. Let $f: \mathbf{C} \rightarrow M$ be a holomorphic curve such that $f(\mathbf{C}) \not \subset D$. Let $\Omega$ be as in (3.4). Then there is a positive constant $C$ independent of $f$ such that

$$
T_{f}(r ; L(\bar{D}))=N\left(r ; f^{*} D\right)+m_{f}(r ; \bar{D})+O(1) \leqq C T_{f}(r ; \Omega)+O(1) .
$$

Lemma 4.2 Let $M, \bar{M}, M_{0}$ be as above. Let $L \rightarrow \bar{M}$ be a line bundle on $\bar{M}$. Then there exist a divisor $E$ with $\operatorname{Supp} E \subset \partial M$ and a line bundle $L_{0} \rightarrow M_{0}$ such that $L \cong$ $L(E) \otimes \bar{\eta}^{*} L_{0}$ (in the sense of bundle isomorphism or linear equivalence); moreover, such $L_{0} \rightarrow M_{0}$ is uniquely determined (up to isomorphism).

Proof. Note that $\bar{\eta}: \bar{M} \rightarrow M_{0}$ is a topologically trivial $\mathbf{P}_{1}(\mathbf{C})^{p}$-bundle over $M_{0}$. Hence by Künneth formula we have

$$
H^{2}(\bar{M}, \mathbf{Z})=H^{2}\left(\mathbf{P}_{1}(\mathbf{C}), \mathbf{Z}\right)^{p} \oplus H^{2}\left(M_{0}, \mathbf{Z}\right)
$$


Since the higher direct image sheaves $\mathcal{R}^{q} \eta_{*} \mathcal{O}, q \geqq 1$, vanish, it follows that $H^{*}(\bar{M}, \mathcal{O}) \cong$ $H^{*}\left(M_{0}, \mathcal{O}\right)$. We deduce that the Picard group $\operatorname{Pic}(\bar{M})$ is generated by $\bar{\eta}^{*} \operatorname{Pic}\left(M_{0}\right)$ and the subgroup of $\operatorname{Pic}(\bar{M})$ generated by the irreducible components of $\partial M=\bar{M} \backslash M$. Thus for $\bar{L} \rightarrow \bar{M}$ there exists a divisor $E$ with $\operatorname{Supp} E \subset \partial M$ such that $L \otimes L(-E) \in \bar{\eta}^{*} \operatorname{Pic}\left(M_{0}\right)$; the assertion follows. Q.E.D.

We denote by

$$
\operatorname{St}(D)=\{x \in M ; x+D=D\}^{0}
$$

the identity component of those $x \in M$ which leaves $D$ invariant by translation. The complex semi-subtorus $\operatorname{St}(D)$ (cf. [NW99]) is called the stabilizer of $D$.

Lemma 4.5 (i) Let $Z$ be a divisor on $\bar{M}$ such that $Z \cap M$ is effective. Let $L_{0} \in \operatorname{Pic}\left(M_{0}\right)$ such that $L(Z) \otimes \bar{\eta}^{*} L_{0}^{-1} \cong L(E)$ with $\operatorname{Supp} E \subset \partial M$. Then $c_{1}\left(L_{0}\right) \geqq 0$.

(ii) Let $D$ be an effective divisor on $M$ with compactification $\bar{D}$ as above. Assume that $\operatorname{St}(D)=\{0\}$. Then $\bar{D}$ is ample on $\bar{M}$.

Proof. (i) Assume the contrary. Recall that $M_{0}$ is a compact complex torus with universal covering $\pi_{0}: \mathbf{C}^{m} \rightarrow M_{0}$. We may regard the Chern class $c_{1}\left(L_{0}\right)$ as bilinear form on the vector space $\mathbf{C}^{m}$. Suppose that $c_{1}\left(L_{0}\right)$ is not semi-positive definite. Let $v \in \mathbf{C}^{m}$ with $c_{1}\left(L_{0}\right)(v, v)<0$ and let $W$ denote the orthogonal complement of $v$ (i.e. $W=\{w \in$ $\left.\left.\mathbf{C}^{m}: c_{1}\left(L_{0}\right)(v, w)=0\right\}\right)$. Let $\mu$ be a semi-positive skew-Hermitian form on $\mathbf{C}^{m}$ such that $\mu(v, \cdot) \equiv 0$ and $\left.\mu\right|_{W \times W}>0$. Now consider the $(n-1, n-1)$-form $\omega$ on $\bar{M}$ given by

$$
\omega=\Omega^{p} \wedge \bar{\eta}^{*} \mu^{m-1}
$$

By construction we have $\omega \wedge \bar{\eta}^{*} c_{1}\left(L_{0}\right)<0$. Let $Z=Z^{\prime}+Z^{\prime \prime}$ so that $Z^{\prime}$ is effective and has no component of $\partial M$, and $\operatorname{Supp} Z^{\prime \prime} \subset \partial M$.

By the Poincaré duality,

$$
\int_{\bar{M}} c_{1}(L(Z)) \wedge \omega=\int_{Z} \omega
$$

Since $\omega \wedge c_{1}(L(E))=0$, we have

$$
\int_{\bar{M}} c_{1}(L(Z)) \wedge \omega=\int_{\bar{M}} \bar{\eta}^{*} c_{1}\left(L_{0}\right) \wedge \omega<0 .
$$

On the other hand,

$$
\int_{Z} \omega=\int_{Z^{\prime}} \omega+\int_{Z^{\prime \prime}} \omega
$$

Note that $\int_{Z^{\prime}} \omega \geqq 0$, because $Z^{\prime}$ is effective and $\omega \geqq 0$, and that $\int_{Z^{\prime \prime}} \omega=0$, because $\operatorname{Supp} Z^{\prime \prime} \subset \partial M$, and $\Omega^{p}$ vanishes on $\partial M$ by construction. Thus we deduced a contradiction. 
(ii) When $p=0$, the assertion is well known ([We58]). Assume $p>0$. Let $\mathbf{C}^{*}$ act on $\bar{M}$ as the $k$-th factor of $\left(\mathbf{C}^{*}\right)^{p} \subset M$. Since $\operatorname{St}(D)=\{0\}$, one infers that there is an orbit whose closure intersects $\bar{D}$ transversally. Hence,

$$
c_{1}(L)=\left(n_{1}, \ldots, n_{p} ; c_{1}\left(L_{0}\right)\right)
$$

in the form described in (4.3) with $n_{1}, \ldots, n_{p}>0$.

Now let us consider $L_{0}$ as in the above (i). By (i) we know that $c_{1}\left(L_{0}\right) \geqq 0$. Assume that there is a vector $v \in \mathbf{C}^{m} \backslash\{O\}$ with $c_{1}\left(L_{0}\right)(v, v)=0$. Then we choose $\mu$ and $\omega$ as in (4.6). Because of the definition we have

$$
\int_{\bar{D}} \omega=0
$$

By the flat connection of the bundle $\eta: M \rightarrow M_{0}$, the vector $v$ is identified as a vector field on $M$. Observe that $\bar{\eta}(\bar{D})=M_{0}$. The construction of $\omega$ and (4.8) imply that $v \in T_{x}(D)$ for all $x \in D$. It follows that the one-parameter subgroup corresponding to $v$ must stabilize $D$; this is a contradiction. Thus $c_{1}\left(L_{0}\right)>0$ if $\operatorname{St}(D)=\{0\}$.

Since all $n_{i}>0$ in (4.7) and $c_{1}\left(L_{0}\right)>0$, it follows that $c_{1}(L(\bar{D}))$ is positive. Thus $\bar{D}$ is ample on $\bar{M}$. Q.E.D.

Corollary 4.9 Let $f: \mathbf{C} \rightarrow M$ and $D$ be as above, and let $\Omega$ be as in (3.4). Assume that $\operatorname{St}(D)=\{0\}$. Then we have the following.

(i) There is a positive constant $C$ such that

$$
C^{-1} T_{f}(r ; \Omega)+O(1) \leqq T_{f}\left(r ; c_{1}(\bar{D})\right) \leqq C T_{f}(r ; \Omega)+O(1)
$$

(ii) $S_{f}(r ; \Omega)=S_{f}\left(r ; c_{1}(\bar{D})\right)$.

The proof is clear.

Remark. $\bar{D}$ may be ample even if $\operatorname{St}(D) \neq\{0\}$. For instance, this happens for the diagonal divisor $D$ in $M=\mathbf{C}^{*} \times \mathbf{C}^{*} \hookrightarrow \bar{M}=\mathbf{P}_{1} \times \mathbf{P}_{1}$.

(b) Boundary condition for $D$. We keep the previous notations. Let

$$
\partial M=\bigcup_{j=1}^{p} B_{j}
$$

be the Whitney stratification of the boundary divisor of $M$ in $\bar{M}$; that is, $B_{j}$ consists of all points $x \in \partial M$ such that the number of irreducible components of $\partial M$ passing $x$ is exactly $j$. Set $B_{0}=M$. A connected component of $B_{j}, 0 \leqq j \leqq p$, is called a stratum of the stratification $\bar{M}=\bigcup_{j=0}^{p} B_{j}$. Observe that $\operatorname{dim} B_{j}=n-j$. 
Note that the holomorphic action of $M$ on $M$ by translations is equivariantly extended to an action on $\bar{M}$, which preserves every stratum of $B_{j}, 0 \leqq j \leqq p$.

Let $D$ be an effective divisor of $M$ which can be extended to a divisor $\bar{D}$ on $\bar{M}$ by taking its topological closure of the support. We consider the following boundary condition for $D$ :

Condition $4.11 \bar{D}$ does not contain any stratum of $B_{p}$.

Note that the strata of $B_{p}$ are minimal.

Lemma 4.12 If condition 4.11 is fulfilled, then

$$
\operatorname{dim} \bar{D} \cap B_{j}<\operatorname{dim} B_{j}=n-j, \quad 0 \leqq \forall j \leqq p .
$$

Proof. Assume the contrary. Then there exists a stratum $S \subset B_{j}$ such that $S \subset \bar{D}$. Clearly the closure $\bar{S}$ of $S$ is likewise contained in $\bar{D}$. But the closure of any stratum contains a minimal stratum, i.e., contains a stratum of $B_{p}$. However, this is in contradiction to condition 4.11. Q.E.D.

Example 4.13 Take a classical case where $M$ is the complement of $n+1$ hyperplanes $H_{j}$ of $\mathbf{P}^{n}(\mathbf{C})$ in general position. Then $M \cong\left(\mathbf{C}^{*}\right)^{n}$. Let $D=H_{n+2}$ be an $(n+2)^{\text {th }}$ hyperplane of $\mathbf{P}^{n}(\mathbf{C})$. Then condition 4.11 is equivalent to that all $H_{j}, 1 \leqq j \leqq n+2$, are in general position.

Next we interpret the boundary condition 4.11 in terms of local defining equations of $\bar{D}$. Take $\sigma \in H^{0}(\bar{M}, L(\bar{D}))$ such that $(\sigma)=\bar{D}$. Suppose that $p>0$. Let $x_{0} \in \partial M \cap \bar{D}$ be an arbitrary point. Let $E$ and $L_{0}$ be as in Lemma 1.2 for $L=L(\bar{D})$. We take an open neighborhood $U$ of $\bar{\eta}\left(x_{0}\right)$ such that the restrictions $\bar{M} \mid U$ and $L_{0} \mid U$ to $U$ are trivialized. Write

$$
x_{0}=\left(u_{0}, x_{0}^{\prime \prime}\right) \in\left(\mathbf{P}^{1}(\mathbf{C})\right)^{p} \times U \cong \bar{M} \mid U .
$$

We take an open neighborhood $V$ of $u_{0}$ such that $V \cong \mathbf{C}^{p} \subset\left(\mathbf{P}^{1}(\mathbf{C})\right)^{p}$ with coordinates $\left(u_{1}, \ldots, u_{p}\right)$. Then $L(\bar{D}) \mid(V \times U)$ is trivial, and hence $\sigma \mid(V \times U)$ is given by a polynomial function

$$
\sigma\left(u, x^{\prime \prime}\right)=\sum_{\text {finite }} a_{l_{1} \cdots l_{p}}\left(x^{\prime \prime}\right) u_{1}^{l_{1}} \cdots u_{p}^{l_{p}}, \quad\left(u, x^{\prime \prime}\right) \in V \times U,
$$

with coefficients $a_{l_{1} \ldots l_{p}}\left(x^{\prime \prime}\right)$ holomorphic in $U$. Since $\bar{D}$ has no component of $\partial M, \sigma\left(u, x^{\prime \prime}\right)$ is not divisible by any $u_{j}$. Set $u_{0}=\left(u_{01}, \ldots, u_{0 p}\right)$. Then, after a change of indices of $u_{i}$ 
one may assume that $u_{01}=\cdots=u_{0 q}=0, u_{0 i} \neq 0,1 \leqq q<i \leqq p$. Expand $\sigma\left(u, x^{\prime \prime}\right)$ and set $\sigma_{1}$ and $\sigma_{2}$ as follows:

$$
\begin{aligned}
\sigma\left(u, x^{\prime \prime}\right) & =\sum_{l_{1}+\cdots+l_{q} \geqq 1} a_{l_{1} \cdots l_{p}}\left(x^{\prime \prime}\right) u_{1}^{l_{1}} \cdots u_{p}^{l_{p}}+\sum_{l_{1}=\cdots=l_{q}=0} a_{0 \cdots 0 l_{q+1} \cdots l_{p}}\left(x^{\prime \prime}\right) u_{q+1}^{l_{1}} \cdots u_{p}^{l_{p}}, \\
\sigma_{1} & =\sum_{l_{1}+\cdots+l_{q} \geqq 1} a_{l_{1} \cdots l_{p}}\left(x^{\prime \prime}\right) u_{1}^{l_{1}} \cdots u_{p}^{l_{p}} \\
\sigma_{2} & =\sum_{l_{1}=\cdots=l_{q}=0} a_{0 \cdots 0 l_{q+1} \cdots l_{p}}\left(x^{\prime \prime}\right) u_{q+1}^{l_{1}} \cdots u_{p}^{l_{p}}
\end{aligned}
$$

We have

Lemma 4.16 Let the notation be as above. Then condition 4.11 is equivalent to that for every $x_{0} \in \partial M, \sigma_{2} \not \equiv 0$.

(c) Regularity of stabilizers. Let $M$ be a complex semi-torus with fixed presentation as in (3.1):

$$
0 \rightarrow G=\left(\mathbf{C}^{*}\right)^{p} \rightarrow M \rightarrow M_{0} \rightarrow 0
$$

Definition. A closed complex Lie subgroup $H$ of $M$ is called regular if there is a subset $I \subset\{1, \ldots, p\}$ such that

$$
G \cap H=\left\{\left(z_{1}, \ldots, z_{p}\right) \in G ; z_{i}=1, \forall i \in I\right\}
$$

Regular subgroups are those compatible with the compactification induced by (4.17). The presentation (4.17) induces in a canonical way such presentations for $H$ and $M / H$.

Lemma 4.18 Let $H$ be a regular Lie subgroup of $M$. Then the quotient mapping $M \rightarrow$ $M / H$ is extended holomorphically in a natural way to the compactification

$$
\bar{M} \stackrel{\bar{H}}{\longrightarrow} \overline{(M / H)}
$$

which is a holomorphic fiber bundle of compact complex manifolds with fiber $\bar{H}$.

We will prove the following proposition.

Proposition 4.19 Let $M$ be a semi-torus with presentation ( 4.17 ) and let $D$ be an effective divisor fulfilling the boundary condition 4.11 . Then there exists a finite unramified covering $\mu^{\prime}: M_{0}^{\prime} \rightarrow M_{0}$ such that $\mathrm{St}\left(\mu^{*} D\right)$ is regular in $M^{\prime}$, where $\mu: M^{\prime} \rightarrow M$ is the finite covering of $M$ induced by $\mu^{\prime}$; i.e., $M^{\prime}=M \times_{M_{0}} M_{0}^{\prime}$. 
Remark. Note that $\mu$ extends holomorphically to the unramified covering of the compactification $\bar{M}, \bar{\mu}: \bar{M}^{\prime} \rightarrow \bar{M}$.

Proof. First, if $D$ is invariant under one of the $p$ direct factors of $G=\left(\mathbf{C}^{*}\right)^{p}$ in (4.17), we take the corresponding quotient. Thus we may assume that $\operatorname{St}(D) \cap G$ does not contain anyone of the $p$ coordinate factors of $G$.

Assume that $\operatorname{dim} \operatorname{St}(D) \cap G>0$. Let $I$ be a subgroup of $\operatorname{St}(D) \cap G$ isomorphic to $\mathbf{C}^{*}$. Then there are integers $n_{1}, \ldots, n_{p}$ such that

$$
I=\left\{\left(t^{n_{1}}, \ldots, t^{n_{p}}\right): t \in \mathbf{C}^{*}\right\} .
$$

By re-arranging indices and coordinate changes of type, $z_{i} \mapsto \frac{1}{z_{i}}$, we may assume that there is a natural number $q$ such that $n_{i}>0$ for $i \leqq q$ and $n_{i}=0$ for $i>q$. Let $G=G_{1} \times G_{2}$ with

$$
\begin{aligned}
& G_{1}=\left\{(u_{1}, \ldots, u_{q-1}, \underbrace{1, \ldots, 1}_{p-q}) ; u_{i} \in \mathbf{C}^{*}\right\} \subset G, \\
& G_{2}=\left\{(\underbrace{1, \ldots, 1}_{q}, u_{q+1}, \ldots, z_{p}) ; u_{i} \in \mathbf{C}^{*}\right\} \subset G .
\end{aligned}
$$

Then $I \subset G_{1}$. Consider $\lambda: M \rightarrow M / G_{1}$. If $\lambda(D) \neq M / G_{1}$, then $D$ would be $G_{1}$-invariant and in particular would be invariant under the coordinate factor groups contained in $G_{1}$. Since this was ruled out, we have $\lambda(D)=M / G_{1}$. Now observe that for every $u=\left(u_{1}, \ldots, u_{p}\right) \in \mathbf{C}^{p} \subset\left(\mathbf{P}^{1}(\mathbf{C})\right)^{p}$ we have

$$
\lim _{t \rightarrow 0}\left(t^{n_{1}}, \ldots, t^{n_{p}}\right) \cdot u=\left(0, \ldots, 0, u_{q+1}, u_{q+2}, \ldots, u_{p}\right) .
$$

Hence it follows from $I \subset \operatorname{St}(D)$ and $\lambda(D)=M / G_{1}$ that

$$
\{0\}^{q} \times\left(\mathbf{P}^{1}(\mathbf{C})\right)^{p-q} \subset \bar{D} .
$$

This violates the boundary condition 4.11 because of Lemma 4.12. Thus $G \cap \operatorname{St}(D)$ is zero-dimensional, and hence finite. As a consequence, $\operatorname{St}(D)$ is compact. After a finite covering, $\operatorname{St}(D)$ maps injectively in $M_{0}$ and therefore is regular. Q.E.D.

\section{Proof of the Main Theorem.}

We first prove the following key lemma:

Lemma 5.1 Assume the same conditions as in the Main Theorem. Then,

$$
m_{f}(r ; \bar{D})=S_{f}\left(r ; c_{1}(\bar{D})\right) .
$$


Besides the conditions stated above, we may also assume by Proposition 4.19 and Lemma 4.5, (ii) that $\operatorname{St}(D)=\{0\}, \bar{D}$ is ample on $\bar{M}$, and hence $M$ is a semi-Abelian variety $A$ :

$$
0 \rightarrow\left(\mathbf{C}^{*}\right)^{p} \rightarrow A \rightarrow A_{0} \rightarrow 0 .
$$

We keep these throughout in this section.

Here we need the notion of logarithmic jet spaces due to [No86]. Since $\partial A$ has only normal crossings, we have the logarithmic $k$-th jet bundle $J_{k}(\bar{A} ; \log \partial A)$ over $\bar{A}$ along $\partial A$, and a morphism

$$
\psi_{k}: J_{k}(\bar{A} ; \log \partial A) \rightarrow J_{k}(\bar{A})
$$

such that the sheaf of germs of holomorphic sections of $J_{k}(\bar{A} ; \log \partial A)$ is isomorphic to that of logarithmic $k$-jet fields (see [No86], Proposition (1.15); there, a "subbundle" $J_{k}(\bar{A} ; \log \partial A)$ of $J_{k}(\bar{A})$ should be understood in this way). Because of the flat structure of the logarithmic tangent bundle $\mathbf{T}(\bar{A} ; \log \partial A)$,

$$
J_{k}(\bar{A} ; \log \partial A) \cong \bar{A} \times \mathbf{C}^{n k} .
$$

Let

$$
\begin{aligned}
& \pi_{1}: J_{k}(\bar{A} ; \log \partial A) \cong \bar{A} \times \mathbf{C}^{n k} \rightarrow \bar{A}, \\
& \pi_{2}: J_{k}(\bar{A} ; \log \partial A) \cong \bar{A} \times \mathbf{C}^{n k} \rightarrow \mathbf{C}^{n k}
\end{aligned}
$$

be the first and the second projections. For a $k$-jet $y \in J_{k}(\bar{A} ; \log \partial A)$ we call $\pi_{2}(y)$ the jet part of $y$.

Let $x \in \bar{D}$ and let $\sigma=0$ be a local defining equation of $\bar{D}$ about $x$. For a germ $g:(\mathbf{C}, 0) \rightarrow(A, x)$ of a holomorphic mapping we denote its $k$-jet by $j_{k}(g)$ and write

$$
d^{j} \sigma(g)=\left.\frac{d^{j}}{d \zeta^{j}}\right|_{\zeta=0} \sigma(g(\zeta)) .
$$

We set

$$
\begin{aligned}
J_{k}(\bar{D})_{x} & =\left\{j_{k}(g) \in J_{k}(\bar{A})_{x} ; d^{j} \sigma(g)=0,1 \leqq j \leqq k\right\}, \\
J_{k}(\bar{D}) & =\bigcup_{x \in D} J_{k}(\bar{D})_{x}, \\
J_{k}(\bar{D} ; \log \partial A) & =\psi_{k}^{-1} J_{k}(\bar{D}) .
\end{aligned}
$$

Then $J_{k}(\bar{D} ; \log \partial A)$ is a subspace of $J_{k}(\bar{A} ; \log \partial A)$, which is depending in general on the embedding $\bar{D} \hookrightarrow \bar{A}$ (cf. [No86]). Note that $\pi_{2}\left(J_{k}(\bar{D} ; \log \partial A)\right)$ is an algebraic subset of $\mathbf{C}^{n k}$. 
Let $J_{k}(f): \mathbf{C} \rightarrow J_{k}(\bar{A} ; \log \partial A)=\bar{A} \times \mathbf{C}^{n k}$ be the $k$-th jet lifting of $f$. Then by [No98] the Zariski closure of $J_{k}(f)(\mathbf{C})$ in $J_{k}(\bar{A} ; \log \partial A)$ is of the form, $\bar{A} \times W_{k}$, with an affine irreducible subvariety $W_{k} \subset \mathbf{C}^{n k}$. Let $\pi: \mathbf{C}^{n} \rightarrow A$ be the universal covering and let

$$
\tilde{f}: z \in \mathbf{C} \rightarrow\left(\tilde{f}_{1}(z), \ldots, \tilde{f}_{n}(z)\right) \in \mathbf{C}^{n}
$$

be the lifting of $f$. Assume that $f$ is of finite order. Then $\tilde{f}(z)$ is a vector valued polynomial by Lemma 3.9. Note that every non-constant polynomial map from $\mathbf{C}$ to $\mathbf{C}^{n}$ is proper, and hence the image is an algebraic subset. It follows that

$$
W_{k}=\overline{\left\{\left(\tilde{f}^{\prime}(z), \ldots, \tilde{f}^{(k)}(z)\right) z \in \mathbf{C}\right\}}=\left\{\left(\tilde{f}^{\prime}(z), \ldots, \tilde{f}^{(k)}(z)\right), z \in \mathbf{C}\right\},
$$

and hence $\operatorname{dim} W_{k} \leqq 1$. Thus we deduced the following lemma.

Lemma 5.3 Let the notation be as above. If $f: \mathbf{C} \rightarrow A$ is of finite order, then $\operatorname{dim} W_{k} \leqq$ 1 and for every point $w_{k} \in W_{k}$ there is a point $a \in \mathbf{C}$ with $\pi_{2} \circ J_{k}(f)(a)=w_{k}$.

Lemma 5.4 Let the notation be as above.

(i) Suppose that $f$ is of finite order $\rho_{f}$. Then there is a number $k_{0}=k_{0}\left(\rho_{f}, D\right)$ such that

$$
\pi_{2}\left(J_{k}(\bar{D} ; \log \partial A)\right) \cap W_{k}=\emptyset, \quad k \geqq k_{0} .
$$

(ii) Suppose that $f$ is of infinite order. Then there is a number $k_{0}=k_{0}(f, D)$ such that

$$
\pi_{2}\left(J_{k}(\bar{D} ; \log \partial A)\right) \cap W_{k} \neq W_{k}, \quad k \geqq k_{0} .
$$

Proof. (i) By making use of (5.2) we have the projection $p_{k, l}: \mathbf{C}^{n k} \rightarrow \mathbf{C}^{n l}$ for $k \geqq l$ induced from the canonical projection $J_{k}(\bar{A} ; \log \partial A) \rightarrow J_{l}(\bar{A} ; \log \partial A)$. For a subset or a point $E_{k}$ of $\mathbf{C}^{n k}$ and $l \leqq k$ we write $E_{k, l}=p_{k, l}\left(E_{k}\right)$.

We see first by Lemma 3.9 that $\rho_{f} \in \mathbf{Z}$, and $\tilde{f}(z)$ is a vector valued polynomial of order $\leqq \rho_{f}$. Thus, $W_{k}$ is of form

$$
W_{k}=(W_{k, \rho_{f}}, \underbrace{O, \ldots, O}_{k-\rho_{f}}) .
$$

Set $W_{k}^{\prime}=W_{k} \cap \pi_{2}\left(J_{k}(\bar{D} ; \log \partial A)\right)$. Then we have

$$
W_{k}^{\prime}=(W_{k, \rho_{f}}^{\prime}, \underbrace{O, \ldots, O}_{k-\rho_{f}}) .
$$


Assume that the present assertion fails. Then, by the Noetherian property of algebraic subsets, there is a point $\xi_{\rho_{f}} \in \bigcap_{k=\rho_{f}}^{\infty} W_{k, \rho_{f}}^{\prime}$ such that, setting $\xi_{k}=(\xi_{\rho_{f}}, \underbrace{O, \ldots, O}_{k-\rho_{f}}) \in \mathbf{C}^{n k}$, we have

$$
\xi_{k} \in \pi_{2}\left(J_{k}(\bar{D} ; \log \partial A)\right), \quad \forall k \geqq \rho_{f} .
$$

We identify $\xi_{k}$ with a logarithmic $k$-jet field on $\bar{A}$ along $\partial A$ (see [No86]). Set $S_{k}=$ $\pi_{1}\left(J_{k}(\bar{D} ; \log \partial A) \cap \pi_{2}^{-1}\left(\xi_{k}\right)\right)$. Then,

$$
\bar{D} \supset S_{\rho_{f}} \supset S_{\rho_{f}+1} \supset \cdots,
$$

which stabilize to $S_{0}=\bigcap_{k=\rho_{f}}^{\infty} S_{k} \neq \emptyset$. Let $x_{0} \in S_{0}$. If $x_{0} \in A$, it follows from 5.3 that there are points $a \in \mathbf{C}$ and $y_{0} \in A$ such that

$$
\begin{aligned}
f(a)+x_{0}+y_{0} & \in D, \\
\left.\frac{d^{k}}{d z^{k}}\right|_{z=a} \sigma(f(z)) & =0, \quad \forall k \geqq 1,
\end{aligned}
$$

where $\sigma$ is a local defining function of $D$ about $f(a)+x_{0}+y_{0}$. Therefore

$$
f(\mathbf{C})+x_{0}+y_{0} \subset D
$$

and hence this contradicts the Zariski denseness of $f(\mathbf{C})$ in $A$. This finishes the proof in the case of $x_{0} \in A$.

Suppose now that $x_{0} \in \bar{A} \backslash A$. Let $\partial A=\bigcup B_{j}$ be the Whitney stratification as in (4.10), and let $x_{0} \in B_{q}$. Let $B$ be the stratum of $B_{q}$ containing $x_{0}$. Then $B$ itself is a semi-Abelian variety such that

$$
0 \rightarrow\left(\mathbf{C}^{*}\right)^{p-q} \rightarrow B \rightarrow A_{0} \rightarrow 0
$$

Let $\sigma\left(u, x^{\prime \prime}\right)=\sigma_{1}\left(u, x^{\prime \prime}\right)+\sigma_{2}\left(u, x^{\prime \prime}\right)$ be as in (4.15) and define $\bar{D}$ in a neighborhood $W$ of $x_{0}$ such that $W$ is of type $V \times U$ as in (4.14). It follows from Lemma 4.16 that $\sigma_{2} \not \equiv 0$. Note that $\bar{D} \cap W \cap B$ is defined by $\sigma_{2}=0$ in $B$. There is a point $a \in \mathbf{C}$ such that $\pi_{2} \circ J_{\rho_{f}}(f)(a)=\xi_{\rho_{f}}$. Dividing the coordinates into three blocks, we set

$$
x_{0}=(\underbrace{0, \ldots, 0}_{q}, x_{0}^{\prime}, x_{0}^{\prime \prime}) \text {. }
$$

We may regard $w_{0}=\left(x_{0}^{\prime}, x_{0}^{\prime \prime}\right) \in B$. Taking a shift $f(z)+y_{0}$ with $y_{0} \in A$ so that $f(a)+y_{0} \in W$, we set in a neighborhood of $a \in \mathbf{C}$

$$
\begin{aligned}
f(z)+y_{0} & =\left(u_{1}(z), \ldots, u_{q}(z), u_{q+1}(z), \ldots, u_{p}(z), x^{\prime \prime}(z)\right) \in W, \\
g(z) & =\left(u_{q+1}(z), \ldots, u_{p}(z), x^{\prime \prime}(z)\right) \in W \cap B .
\end{aligned}
$$


Here we may choose $y_{0}$ so that $g(a)=w_{0}$.

We set $\xi_{k}=\pi_{2} \circ J_{k}(f)(a)$ for all $k \geqq 1$. Using the same coordinate blocks as (5.5), we set

$$
\begin{aligned}
\xi_{k} & =\left(\xi_{k(1)}^{\prime}, \xi_{k(2)}^{\prime}, \xi_{k}^{\prime \prime}\right), \quad k \geqq \rho_{f}, \\
\xi_{k(2)} & =\text { the jet part of } J_{k}(g)(a)=\left(\xi_{k(2)}^{\prime}, \xi_{k}^{\prime \prime}\right) .
\end{aligned}
$$

Since the logarithmic term (e.g., $z_{j} \frac{\partial}{\partial z_{j}}, 1 \leqq j \leqq q$, in the case of 1 -jets) of a logarithmic jet field vanishes on the corresponding divisor locus (e.g., $\bigcup_{j=1}^{q}\left\{z_{j}=0\right\}$ ) (see [No86], $\S 1$ and (1.14) for more details), we have $\xi_{k}\left(\sigma_{1}\right)\left(x_{0}\right)=0$ by (4.15), and hence $\xi_{k(2)}\left(\sigma_{2}\right)\left(x_{0}\right)=$ $0, \forall k \geqq 1 ;$ i.e.,

$$
\left.\frac{d^{k}}{d z^{k}}\right|_{z=a} \sigma_{2}(g(z))=0, \quad \forall k \geqq 0 .
$$

Let $\left(\mathbf{C}^{*}\right)^{q}$ be the first $q$-factor of the subgroup $\left(\mathbf{C}^{*}\right)^{p} \subset A$, and let $\lambda: A \rightarrow A /\left(\mathbf{C}^{*}\right)^{q} \cong B$ be the quotient map. By (5.5) and (5.6) the composed map $g(z)=\lambda \circ\left(f(z)+y_{0}\right)$ has an image contained in $\bar{D} \cap B$; that is, it has no Zariski dense image in $A /\left(\mathbf{C}^{*}\right)^{q}$, and hence so is $f$; this is a contradiction.

The order of the tangency of $f$ and the above used $g$ with $\bar{D}$ is bounded as $\tilde{f}$ runs over all vector valued polynomials of order at most $\rho_{f}$ such that $f(\mathbf{C})$ is Zariski dense in $A$. Hence there is such a number $k_{0}$ depending only on $\rho_{f}$ and $D$.

(ii) Assume contrarily that $\pi_{2}\left(J_{k}(\bar{D} ; \log \partial A)\right) \cap W_{k}=W_{k}$ for all $k \geqq 1$. Since $\pi_{2} \circ$ $J_{k}(f)(0) \in W_{k}, \forall k \geqq 1$, we apply the same argument as in (i) with setting $\xi_{k}=\pi_{2} \circ$ $J_{k}(f)(0)$. Then we deduce a contradiction that $f$ has no Zariski dense image. Q.E.D.

Proof of Lemma 5.1. For a multiple $l \bar{D}$ of $\bar{D}$ we have

$$
m_{f}(r ; l \bar{D})=l m_{f}(r ; \bar{D})
$$

Thus we may assume that $\bar{D}$ is very ample on $\bar{A}$. Let $\left\{\tau_{j}\right\}_{j=1}^{N}$ be a base of $H^{0}(\bar{A}, L(\bar{D}))$ such that $\operatorname{Supp}\left(\tau_{j}\right) \not \supset f(\mathbf{C})$ for all $1 \leqq j \leqq N$. Since $\bar{D}$ is very ample, the sections $\tau_{j}$, $1 \leqq j \leqq N$, have no common zero. Set

$$
U_{j}=\left\{\tau_{j} \neq 0\right\}, \quad 1 \leqq j \leqq N
$$

Then $\left\{U_{j}\right\}$ is an affine open covering of $\bar{A}$. Let $\sigma \in H^{0}(\bar{A}, L(\bar{D}))$ be a section such that $(\sigma)=\bar{D}$. We define a regular function $\sigma_{j}$ on every $U_{j}$ by

$$
\sigma_{j}(x)=\frac{\sigma(x)}{\tau_{j}(x)} .
$$


Note that $\sigma_{j}$ is a defining function of $\bar{D} \cap U_{j}$. Let us now fix a hermitian metric $\|\cdot\|$ on $L(\bar{D})$. Then there are positive smooth functions $h_{j}$ on $U_{j}$ such that

$$
\frac{1}{\|\sigma(x)\|}=\frac{h_{j}(x)}{\left|\sigma_{j}(x)\right|}, \quad x \in U_{j}
$$

Assume that $f$ is of finite order. By Lemma 5.4 there are regular functions $b_{j i}, 0 \leqq i \leqq$ $k_{0}$, on $U_{j} \times W_{k_{0}}$ such that

$$
b_{j 0} \sigma_{j}+b_{j 1} d \sigma_{j}+\cdots+b_{j k_{0}} d^{k_{0}} \sigma_{j}=1 .
$$

Here every $b_{j i}$ is expressed as

$$
b_{j i}=\sum_{\text {finite }} b_{j i l \beta_{l}}(x) w_{l}^{\beta_{l}}
$$

where $b_{i \alpha j l \beta_{l}}(x)$ are regular functions on $U_{j}$ and $w_{l}$ are restrictions of coordinate functions of $\mathbf{C}^{n k_{0}}$ to $W_{k_{0}}$. Thus we infer that in every $U_{j}$

$$
\frac{1}{\|\sigma\|}=\frac{h_{j}}{\left|\sigma_{j}\right|}=\left|h_{j} b_{j 0}+h_{j} b_{j 1} \frac{d \sigma_{j}}{\sigma_{j}}+\cdots+h_{j} b_{j k_{0}} \frac{d^{k_{0}} \sigma_{j}}{\sigma_{j}}\right| .
$$

Take relatively compact open subsets $U_{j}^{\prime} \Subset U_{j}$ (in the sense of differential topology) so that $\bigcup U_{j}^{\prime}=\bar{A}$. For every $j$ there is a positive constant $C_{j}$ such that for $x \in U_{j}^{\prime}$

$$
h_{j}\left|b_{j i}\right| \leqq \sum_{\text {finite }} h_{j}\left|b_{j i l \beta_{l}}(x)\right| \cdot\left|w_{l}\right|^{\beta_{l}} \leqq C_{j} \sum_{\text {finite }}\left|w_{l}\right|^{\beta_{l}} .
$$

Thus, after making $C_{j}$ larger if necessary, there is a number $d_{j}>0$ such that for $f(z) \in U_{j}^{\prime}$

$$
h_{j}(f(z))\left|b_{j i}\left(J_{k_{0}}(f)(z)\right)\right| \leqq C_{j}\left(1+\sum_{1 \leqq l \leqq n, 1 \leqq k \leqq k_{0}}\left|\tilde{f}_{l}^{(k)}(z)\right|\right)^{d_{j}}
$$

We deduce that

$$
\begin{aligned}
\frac{1}{\|\sigma(f(z))\|} \leqq & \sum_{j=1}^{N} C_{j}\left(1+\sum_{1 \leqq l \leqq n, 1 \leqq k \leqq k_{0}}\left|\tilde{f}_{l}^{(k)}(z)\right|\right)^{d_{j}} \\
& \times\left(1+\left|\frac{d \sigma_{j}}{\sigma_{j}}\left(J_{1}(f)(z)\right)\right|+\cdots+\left|\frac{d^{k_{0}} \sigma_{j}}{\sigma_{j}}\left(J_{k_{0}}(f)(z)\right)\right|\right) .
\end{aligned}
$$

Hence one gets

$$
\begin{aligned}
m_{f}(r ; \bar{D})= & \frac{1}{2 \pi} \int_{0}^{2 \pi} \log ^{+} \frac{1}{\left\|\sigma\left(f\left(r e^{i \theta}\right)\right)\right\|} d \theta \\
\leqq & O\left(\sum_{1 \leqq l \leqq n, 1 \leqq k \leqq k_{0}} \frac{1}{2 \pi} \int_{0}^{2 \pi} \log ^{+}\left|\tilde{f}_{l}^{(k)}\left(r e^{i \theta}\right)\right| d \theta\right. \\
& \left.+\sum_{1 \leqq j \leqq N, 1 \leqq k \leqq k_{0}} \frac{1}{2 \pi} \int_{0}^{2 \pi} \log ^{+}\left|\frac{d^{k} \sigma_{j}}{\sigma_{j}}\left(J_{k}(f)\left(r e^{i \theta}\right)\right)\right| d \theta\right)+O(1) .
\end{aligned}
$$


Recall that the rational functions $\sigma_{j}$ are equal to quotients of two holomorphic sections $\sigma$ and $\tau_{j}$ of $L(\bar{D})$. By Lemma 2.5, (ii) we see that

$$
\frac{1}{2 \pi} \int_{0}^{2 \pi} \log ^{+}\left|\frac{d^{k} \sigma_{j}}{\sigma_{j}}\left(J_{k}(f)\left(r e^{i \theta}\right)\right)\right| d \theta=m\left(r, \frac{\left(\sigma_{j} \circ f\right)^{(k)}}{\sigma_{j} \circ f}\right)=O(\log r) .
$$

This combined with (5.9) and Lemma 3.8 implies that $m_{f}(r ; \bar{D})=O(\log r)$; this completes the proof in the case of finite order.

Assume that $f$ is of infinite order. It follows from Lemma 5.4, (ii) that there exists a polynomial function $R(w)$ in $w \in W_{k_{0}}$ such that

$$
\pi_{2}\left(J_{k}(\bar{D} ; \log \partial A)\right) \cap W_{k} \subset\left\{w \in W_{k_{0}} ; R(w)=0\right\} \neq W_{k_{0}} .
$$

We regard $R$ as a regular function on every $U_{j} \times W_{k_{0}}$. Then we have the following equation on every $U_{j} \times W_{k_{0}}$ with coefficients similar to those of (5.7):

$$
b_{j 0} \sigma_{j}+b_{j 1} d \sigma_{j}+\cdots+b_{j k_{0}} d^{k_{0}} \sigma_{j}=R .
$$

Then, after the same arguments as in the case of finite order, we have that for $f(z) \in U_{j}^{\prime}$

$$
\begin{aligned}
\frac{1}{\|\sigma(f(z))\|}= & \frac{1}{\left|R\left(\tilde{f}^{\prime}(z), \ldots, \tilde{f}\left(k_{0}\right)(z)\right)\right|} \\
& \times\left|h_{j} b_{j 0}+h_{j} b_{j 1} \frac{d \sigma_{j}}{\sigma_{j}}+\cdots+h_{j} b_{j k_{0}} \frac{d^{k_{0}} \sigma_{j}}{\sigma_{j}}\right| \\
\leqq & \frac{1}{\left|R\left(\tilde{f}^{\prime}(z), \ldots, \tilde{f}\left(k_{0}\right)(z)\right)\right|} \sum_{j^{\prime}=1}^{N} C_{j^{\prime}}\left(1+\sum_{1 \leqq l \leqq n, 1 \leqq k \leqq k_{0}}\left|\tilde{f}_{l}^{(k)}(z)\right|\right)^{d_{j^{\prime}}} \\
& \times\left(1+\left|\frac{d \sigma_{j^{\prime}}}{\sigma_{j^{\prime}}}\left(J_{1}(f)(z)\right)\right|+\cdots+\left|\frac{d^{k_{0}} \sigma_{j^{\prime}}}{\sigma_{j^{\prime}}}\left(J_{k_{0}}(f)(z)\right)\right|\right) .
\end{aligned}
$$

It follows that

$$
\begin{aligned}
m_{f}(r ; \bar{D}) \leqq & \frac{1}{2 \pi} \int_{0}^{2 \pi} \log ^{+} \frac{1}{\left\|\sigma\left(f\left(r e^{i \theta}\right)\right)\right\|} d \theta+O(1) \\
\leqq & m\left(r, \frac{1}{R\left(\tilde{f}^{\prime}, \ldots, \tilde{f}^{\left(k_{0}\right)}\right)}\right)+O\left(\sum_{1 \leqq l \leqq n, 1 \leqq k \leqq k_{0}} m\left(r, \tilde{f}_{l}^{(k)}\right)\right. \\
& \left.+\sum_{1 \leqq j \leqq N, 1 \leqq k \leqq k_{0}} m\left(r, \frac{d^{k} \sigma_{j}}{\sigma_{j}} \circ J_{k}(f)\right)\right)+O(1) \\
\leqq & T\left(r, R\left(\tilde{f}^{\prime}, \ldots, \tilde{f}^{\left(k_{0}\right)}\right)\right) \\
& +O\left(\sum_{l, k, j} m\left(r, \tilde{f}_{j}^{(k)}\right)+m\left(r, \frac{\left(\sigma_{j} \circ f\right)^{(k)}}{\sigma_{j} \circ f}\right)\right)+O(1) .
\end{aligned}
$$


This combined with Lemmas 3.8 and 2.5 implies that $m_{f}(r ; \bar{D})=S_{f}\left(r ; c_{1}(\bar{D})\right)$. This finishes the proof. Q.E.D.

Proof of the Main Theorem. We keep the notation used above. Thanks to Lemma 5.1 the only things we still have to show are the statements on the truncation, i.e., the bounds on $N\left(r ; f^{*} D\right)-N_{k_{0}}\left(r ; f^{*} D\right)$. Observe that $\operatorname{ord}_{z} f^{*} D>k$ if and only if $J_{k}(f)(z) \in$ $\left.J_{k}(\bar{D} ; \log \partial A)\right)$. Therefore, if $f$ is of finite order, Lemma 5.4, (i) implies that $N\left(r ; f^{*} D\right)=$ $N_{k_{0}}\left(r ; f^{*} D\right)$.

In the case where $f$ is of infinite order we infer from (5.11) that

$$
\operatorname{ord}_{z} f^{*} D-\min \left\{\operatorname{ord}_{z} f^{*} D, k_{0}\right\} \leqq \operatorname{ord}_{z}\left(R\left(\tilde{f}^{\prime}, \ldots, \tilde{f}^{k_{0}}\right)\right)_{0} .
$$

Thus we have after integration that

$$
N\left(r ; f^{*} D\right)-N_{k_{0}}\left(r ; f^{*} D\right) \leqq N\left(r ;\left(R\left(\tilde{f}^{\prime}, \ldots, \tilde{f}^{\left(k_{0}\right)}\right)\right)_{0}\right) .
$$

It follows from (2.2), (2.3) and Lemma 3.8 that

$$
\begin{aligned}
N\left(r ;\left(R\left(\tilde{f}^{\prime}, \ldots, \tilde{f}^{\left(k_{0}\right)}\right)\right)_{0}\right) & \leqq T\left(r, R\left(\tilde{f}^{\prime}, \ldots, \tilde{f}^{\left(k_{0}\right)}\right)\right)+O(1) \\
& \leqq O\left(\sum_{1 \leqq l \leqq n, 1 \leqq k \leqq k_{0}} T\left(r, \tilde{f}_{l}^{(k)}\right)\right) \\
& =S_{f}(r ; \Omega) .
\end{aligned}
$$

Furthermore, $S_{f}(r ; \Omega)=S_{f}\left(r, c_{1}(D)\right)$ by Corollary 4.9, (ii), because $\bar{D}$ is ample. Hence,

$$
N\left(r ; f^{*} D\right) \leqq N_{k_{0}}\left(r ; f^{*} D\right)+S_{f}\left(r ; c_{1}(\bar{D})\right)
$$

The proof is completed. Q.E.D.

By (3.5), (3.9) and Lemma 5.1 we have

Corollary 5.12 Let $M$ be a complex torus and let $f: \mathbf{C} \rightarrow M$ be an arbitrary holomorphic curve. Let $D$ be an effective divisor on $M$ such that $D \not \supset f(\mathbf{C})$. Then we have the following.

(i) Suppose that $f$ is of finite order $\rho_{f}$. Then there is a positive integer $k_{0}=k_{0}\left(\rho_{f}, D\right)$ such that

$$
T_{f}\left(r ; c_{1}(D)\right)=N_{k_{0}}\left(r ; f^{*} D\right)+O(\log r) .
$$

(ii) Suppose that $f$ is of infinite order. Then there is a positive integer $k_{0}=k_{0}(f, D)$ such that

$$
T_{f}\left(r ; c_{1}(D)\right)=N_{k_{0}}\left(r ; f^{*} D\right)+S_{f}\left(r ; c_{1}(D)\right) .
$$


Specially, $\delta(f ; D)=\delta_{k_{0}}(f ; D)=0$ in both cases.

Proof. Since the Zariski closure of $f(\mathbf{C})$ is a translation of a complex subtorus of $M$ (cf., e.g., [NO $\left.\frac{84}{90}\right]$, Chap. VI, [Ko98], Chap. 3, §9, [NW99]), we may assume that $f(\mathbf{C})$ is Zariski dense. Hence this statement is a special case of the Main Theorem. Q.E.D.

Proposition 5.13 Let $M$ be a complex semi-torus $M$ and let $D$ be an effective divisor on $M$ such that its topological closure $\bar{D}$ is a divisor in $\bar{M}$. Assume that $D$ violates the boundary condition 4.11. Then there exists an entire holomorphic curve $f: \mathbf{C} \rightarrow M$ of an arbitrarily given integral order $\rho \geqq 2$ in general, and $\rho \geqq 1$ in the case of $M_{0}=\{0\}$ such that $f(\mathbf{C})$ is Zariski dense in $M$ and $\delta(f ; \bar{D})>0$.

Proof. Let $\hat{M}=\left(\mathbf{P}^{1}(\mathbf{C})\right)^{p} \times \mathbf{C}^{m} \rightarrow \bar{M}$ (resp. $\mathbf{C}^{m} \rightarrow M_{0}$ ) be the universal covering of $\bar{M}$ (resp. $M_{0}$ ), and $\hat{D} \subset \hat{M}$ the preimage of $\bar{D}$. We may assume that

$$
\{(\infty)\}^{p} \times \mathbf{C}^{m} \subset \hat{D} .
$$

Let $c_{1}, \ldots, c_{p}$ be $\mathbf{Q}$-linear independent real numbers with

$$
0<c_{1}<c_{2}<\cdots<c_{p}
$$

Let $\rho \geqq 2$ or $\rho \geqq 1$ be an arbitrary integer as assumed in the proposition, and set

$$
\hat{f}: z \mapsto\left(\left[1: e^{c_{1} z^{\rho}}\right],\left[1: e^{c_{2} z^{\rho}}\right], \ldots,\left[1: e^{c_{p} z^{\rho}}\right] ; L(z)\right)
$$

where $L: \mathbf{C} \rightarrow \mathbf{C}^{m}$ is a linear map such that the image $L(\mathbf{C})$ in $M_{0}$ is Zariski dense. Moreover, by a generic choice of $c_{j}$ and $L$ we have that $f(\mathbf{C})$ is Zariski dense in $M$. Let

$$
U_{i} \Subset V_{i} \Subset M_{0}
$$

be a finite collection of relatively compact holomorphically convex open subsets of $M_{0}$ such that there are sections $\mu_{i}: V_{i} \stackrel{\sim}{\rightarrow} \hat{V}_{i} \subset \mathbf{C}^{m}$ and such that the $U_{i}$ cover $M_{0}$. Set $\hat{U}_{i}=\mu_{i}\left(U_{i}\right)$.

For every $i$ the restricted divisor $\hat{D} \mid\left(\left(\mathbf{P}^{1}(\mathbf{C})\right)^{p} \times \hat{V}_{i}\right)$ is defined by a homogeneous polynomial $P_{i 0}$ of multidegree $\left(d_{1}, \ldots, d_{p}\right)$, where the coefficients are holomorphic functions on $V_{i}$. Let $P_{i}$ denote the associated inhomogeneous polynomial. Then $P_{i}$ is a polynomial of multidegree $\left(d_{1}, \ldots, d_{p}\right)$. Due to $\{\infty\}^{p} \times \mathbf{C}^{m} \subset \hat{D}, P_{i}$ does not carry the highest degree monomial, $u_{1}^{d_{1}} \cdots u_{p}^{d_{p}}$.

Recall $\bar{M}=\hat{M} / \Lambda_{0}$ where $\Lambda_{0}$ is a lattice in $\mathbf{C}^{m}$ and acts on $\hat{M}$ via

$$
\lambda:\left(u_{1}, \ldots, u_{p} ; x^{\prime \prime}\right) \mapsto \lambda \cdot\left(u ; x^{\prime \prime}\right)=\left(\beta_{1}(\lambda) u_{1}, \ldots, \beta_{p}(\lambda) u_{p} ; x^{\prime \prime}+\lambda\right),
$$

where $\beta: \Lambda_{0} \rightarrow\left(S^{1}\right)^{p}$ is a group homomorphism into the product of $S^{1}=\{|z|=1 ; z \in$ $\left.\mathrm{C}^{*}\right\}$. 
Together with (5.15) and (5.14) this implies that there is a constant $C>0$ such that

$$
\left|P_{i}(\lambda \cdot \hat{f}(z))\right| \leqq C\left|e^{\left(\sum_{j} d_{j} c_{j}\right) z^{\rho}-c_{1} z^{\rho}}\right|
$$

for all $\lambda \in \Lambda_{0}$ and $z \in \mathbf{C}$ with $\Re z^{\rho}>0$ and $\lambda \cdot \hat{f}(z) \in\left(\mathbf{P}^{1}(\mathbf{C})\right)^{p} \times \hat{U}_{i}$. Note that for every $z \in \mathbf{C}$ there exists an element $\lambda \in \Lambda_{0}$ and an index $i$ such that $\lambda \cdot \hat{f}(z) \in\left(\mathbf{P}^{1}(\mathbf{C})\right)^{p} \times \hat{U}_{i}$. Then there is a constant $C^{\prime}>0$ such that

$$
\|\sigma(x)\|^{2} \leqq C^{\prime} \frac{\left|P_{i}(\lambda \cdot x)\right|^{2}}{\prod_{j}\left(1+\left|u_{j}\right|^{2}\right)^{d_{j}}}
$$

for all $x \in \hat{M}, \lambda \in \Lambda_{0}$ with $\lambda \cdot x \in U_{i}$. From (5.16) and (5.17) it follows that for $\Re z^{\rho}>0$

$$
\begin{aligned}
\|\sigma(f(z))\|^{2} & \leqq C^{\prime} C^{2} \frac{\left|e^{\left(\sum_{j} d_{j} c_{j}\right) z^{\rho}-c_{1} z^{\rho}}\right|^{2}}{\prod_{j}\left(1+\left|e^{2 c_{j} z^{\rho}}\right|\right)^{d_{j}}} \leqq C^{\prime} C^{2} \frac{\left|e^{\left(\sum_{j} d_{j} c_{j}\right) z^{\rho}-c_{1} z^{\rho}}\right|^{2}}{\prod_{j}\left|e^{2 c_{j} d_{j} z^{\rho}}\right|} \\
& =C^{\prime} C^{2}\left|e^{-c_{1} z^{\rho}}\right|^{2}=C^{\prime} C^{2} e^{-2 c_{1} \Re z^{\rho}} .
\end{aligned}
$$

Hence,

$$
\log ^{+} \frac{1}{\|\sigma(f(z))\|} \geqq c_{1} \Re z^{\rho}+O(1)
$$

for all $z \in \mathbf{C}$ with $\Re z^{\rho}>0$. Therefore,

$$
\begin{aligned}
m_{f}(r ; \bar{D}) & =\frac{1}{2 \pi} \int_{\{|z|=r\}} \log \frac{1}{\|\sigma(f(z))\|} d \theta \\
& =\frac{1}{2 \pi} \int_{\{|z|=r\}} \log ^{+} \frac{1}{\|\sigma(f(z))\|} d \theta+O(1) \\
& \geqq \frac{1}{2 \pi} \int_{\left\{|z|=r ; \Re z^{\rho}>0\right\}} \log ^{+} \frac{1}{\|\sigma(f(z))\|} d \theta+O(1) \\
& =\frac{1}{2 \pi} \int_{\{|z|=r\}} c_{1} \cdot\left(\Re z^{\rho}\right)^{+} d \theta+O(1) \\
& =\frac{1}{2 \pi} \int_{0}^{2 \pi} c_{1} r^{\rho} \cos ^{+} \rho \theta d \theta+O(1) \\
& =\frac{c_{1}}{\pi} r^{\rho}+O(1) .
\end{aligned}
$$

On the other hand one deduces easily from (5.15) that $T_{f}(r ; D)=O\left(r^{\rho}\right)$. Hence,

$$
\delta(f ; \bar{D})=\varliminf_{r \rightarrow \infty} \frac{m_{f}(r ; \bar{D})}{T_{f}(r ; \bar{D})}>0 .
$$

Q.E.D.

We will now give an explicit example with $\operatorname{St}(D)=\{0\}$. 
Example 5.18 Let $A$ be the semi-abelian variety $A=\mathbf{C}^{*} \times \mathbf{C}^{*}$, compactified by $\mathbf{P}^{1}(\mathbf{C}) \times$ $\mathbf{P}^{1}(\mathbf{C})$ with a pair of homogeneous coordinates, $\left(\left[x_{0}: x_{1}\right],\left[y_{0}: y_{1}\right]\right)$. For a pair of natural numbers $(m, n)$ with $m<n$, let $\bar{D}$ be the divisor given by

$$
\bar{D}=\left\{\left(\left[x_{0}: x_{1}\right],\left[y_{0}: y_{1}\right]\right): y_{0}^{n} x_{1}+y_{0}^{n-m} y_{1}^{m} x_{0}+y_{1}^{n} x_{0}=0\right\} .
$$

Set $D=\bar{D} \cap A$. Note that $\operatorname{St}(D)=\{0\}$. Moreover, $D$ violates condition 4.11, since $\bar{D} \ni([1: 0],[1: 0])$. Let $c$ be a positive irrational real number such that

$$
0<c m<1<c n \text {. }
$$

Let $f: \mathbf{C} \rightarrow A$ be the holomorphic curve given by

$$
f: z \mapsto\left(\left[1: e^{z}\right],\left[1: e^{c z}\right]\right) .
$$

Let $\Omega_{i}, i=1,2$, be the Fubini-Study metric forms of the two factors of $\left(\mathbf{P}^{1}(\mathbf{C})\right)^{2}$. Then $c_{1}(\bar{D})=\Omega_{1}+n \Omega_{2}$. By an easy computation one obtains

$$
T_{f}\left(r ; c_{1}(\bar{D})\right)=\frac{1+n c}{\pi} r+O(1) .
$$

Thus, $\rho_{f}=1$, and the image $f(\mathbf{C})$ is Zariski dense in $A$, because $c$ is irrational.

We compute $N\left(r ; f^{*} D\right)$ as follows. Note the following identity for divisors on $\mathbf{C}$ :

$$
f^{*} D=\left(e^{z}+e^{m c z}+e^{n c z}\right)_{0} .
$$

We consider a holomorphic curve $g$ in $\mathbf{P}^{2}(\mathbf{C})$ with the homogeneous coordinate system $\left[w_{0}: w_{1}: w_{2}\right]$ defined by

$$
g: z \in \mathbf{C} \rightarrow\left[e^{z}: e^{m c z}: e^{n c z}\right] \in \mathbf{P}^{2}(\mathbf{C}) .
$$

By computing the Wronskian of $e^{z}, e^{m c z}$ and $e^{n c z}$ one sees that they are linearly independent over $\mathbf{C}$; that is $g$ is linearly non-degenerate. Let $T_{g}(r)$ be the order function of $g$ with respect to the Fubini-Study metric form on $\mathbf{P}^{2}(\mathbf{C})$. It follows that

$$
\begin{aligned}
T_{g}(r) & =\frac{1}{4 \pi} \int_{\{|z|=r\}} \log \left(\left|e^{z}\right|^{2}+\left|e^{m c z}\right|^{2}+\left|e^{n c z}\right|^{2}\right) d \theta+O(1) \\
& =\frac{1}{4 \pi} \int_{\{|z|=r\}} \log \left(1+\left|e^{(m c-1) z}\right|^{2}+\left|e^{(n c-1) z}\right|^{2}\right) d \theta+O(1) .
\end{aligned}
$$

If $\Re z \geqq 0$ (resp. $\leqq 0),\left|e^{(m c-1) z}\right| \leqq 1$ (resp. $\left.\geqq 1\right)$ and $\left|e^{(n c-1) z}\right| \geqq 1$ (resp. $\leqq 1$ ). Therefore, if $z=r e^{i \theta}$ and $\Re z \geqq 0$,

$$
\begin{aligned}
\log \left(1+\left|e^{(m c-1) z}\right|^{2}+\left|e^{(n c-1) z}\right|^{2}\right) & =2 \log ^{+}\left|e^{(n c-1) z}\right|+O(1) \\
& =2(n c-1) r \cos \theta+O(1) .
\end{aligned}
$$


If $z=r e^{i \theta}$ and $\Re z \leqq 0$,

$$
\begin{aligned}
\log \left(1+\left|e^{(m c-1) z}\right|^{2}+\left|e^{(n c-1) z}\right|^{2}\right) & =2 \log ^{+}\left|e^{(m c-1) z}\right|+O(1) \\
& =2(m c-1) r \cos \theta+O(1) .
\end{aligned}
$$

Combining these with (5.22), we have

$$
T_{g}(r)=\frac{(n-m) c}{\pi} r+O(1)
$$

We consider the following four lines $H_{j}, 1 \leqq j \leqq 4$, of $\mathbf{P}^{2}(\mathbf{C})$ in general position:

$$
H_{j}=\left\{w_{j-1}=0\right\}, \quad 1 \leqq j \leqq 3, \quad H_{4}=\left\{w_{0}+w_{1}+w_{2}=0\right\}
$$

Noting that $g$ is linearly non-degenerate and has a finite order (in fact, $\rho_{g}=1$ ), we infer from Cartan's S.M.T. [Ca33] that

$$
T_{g}(r) \leqq \sum_{j=1}^{4} N_{2}\left(r ; g^{*} H_{j}\right)+O(\log r)
$$

Since $N_{2}\left(r ; g^{*} H_{j}\right)=0,1 \leqq j \leqq 3$, we deduce from (5.24), (5.23) and (2.1) that

$$
N\left(r ; g^{*} H_{4}\right)=\frac{(n-m) c}{\pi} r+O(\log r) .
$$

By $(5.21), N\left(r ; g^{*} H_{4}\right)=N\left(r ; f^{*} D\right)$, and so

$$
N\left(r ; f^{*} D\right)=\frac{(n-m) c}{\pi} r+O(\log r)
$$

It follows from $(5.20)$ and 5.25$)$ that

$$
\delta(f ; \bar{D})=\frac{1+m c}{1+n c} .
$$

By elementary calculations one shows that $\operatorname{ord}_{z} f^{*} D \geqq 2$ implies

$$
(m c-1)\left(e^{c z}\right)^{m}+(n c-1)\left(e^{c z}\right)^{n}=0
$$

Furthermore, $f(z) \in D$ if and only if $e^{z}+e^{m c z}+e^{n c z}=0$. Combined, these two relations imply that there is a finite subset $S \subset \mathbf{C}^{2}$ such that $\operatorname{ord}_{z} f^{*} D \geqq 2 \operatorname{implies}\left(e^{z}, e^{c z}\right) \in S$. Since $z \mapsto\left(e^{z}, e^{c z}\right)$ is injective, it follows that $\left\{z: \operatorname{ord}_{z} f^{*} D \geqq 2\right\}$ is a finite set. Therefore,

$$
\begin{aligned}
N_{1}\left(r, f^{*} D\right) & =N\left(r, f^{*} D\right)+O(\log r), \\
\delta_{1}(f ; \bar{D}) & =\delta(f ; \bar{D})=\frac{1+m c}{1+n c} .
\end{aligned}
$$


Let $c^{\prime}>1$ be an irrational number, and set

$$
c=1 / c^{\prime}, \quad m=\left[c^{\prime}\right], \quad n=\left[c^{\prime}\right]+1,
$$

where $\left[c^{\prime}\right]$ denotes the integral part of $c^{\prime}$. Then $m, n$ and $c$ satisfy (5.19), and by (5.26)

$$
\delta(f ; \bar{D})=\frac{1+\left[c^{\prime}\right] / c^{\prime}}{1+\left(\left[c^{\prime}\right]+1\right) / c^{\prime}} \rightarrow 1 \quad\left(c^{\prime} \rightarrow \infty\right) .
$$

Thus $\delta(f ; \bar{D})\left(=\delta_{1}(f ; \bar{D})\right.$ by $\left.(5.27)\right)$ takes values arbitrarily close to 1 .

Remark 5.28 In [No96], the first author proved that for $D$ without condition 4.11 a holomorphic curve $f: \mathbf{C} \rightarrow A$, omitting $D$, has no Zariski dense image, and is contained in a translate of a semi-Abelian subvariety which has no intersection with $D$. What was proved in [No96] applied to $f: \mathbf{C} \rightarrow A$ with Zariski dense image yields that there is a positive constant $\kappa$ such that

$$
\kappa T_{f}\left(r ; c_{1}(\bar{D})\right) \leqq N_{1}\left(r ; f^{*} D\right)+S_{f}\left(r ; c_{1}(\bar{D})\right)
$$

provided that $\operatorname{St}(D)=\{0\}$. The above $\kappa$ may be, in general, very small because of the method of the proof. One needs more detailed properties of $J_{k}(D)$ to get the best bound such as in the Main Theorem than to get (5.29); this is the reason why we need the boundary condition 4.11 for $D$.

Remark 5.30 In [SiY97] Siu and Yeung claimed (1.2) for Abelian $A$ of dimension $n$. The most essential part of their proof was Lemma 2 of [SiY97], but the claimed assertion does not hold. We recall the lemma.

Lemma 2 ([SiY97], p. 1147). Let $A$ be an n-dimensional Abelian variety and let $D$ be an ample divisor on $A$. Let $f: \mathbf{C} \rightarrow A$ be a holomorphic curve with Zariski dense image, and define $W_{k}$ as in Lemma 5.4. Let $\delta \geqq 1$ and $k \geqq n$ be arbitrarily fixed integers. Then for an arbitrary positive integer $q$ there exists a positive integer $m_{0}\left(W_{k}, \delta, q\right)$ depending on $W_{k}, \delta, q$ (and $A$ and $D)$ such that for $m \geqq m_{0}\left(W_{k}, \delta, q\right)$ there exists an $(L(D))^{\delta}$-valued holomorphic $k$-jet differential $\omega$ on $A$ of weight $m$ whose restriction to $A \times W_{k}$ is not identically zero and which vanishes along $J_{k}(D) \cap\left(A \times W_{k}\right)$ to order at least $q$. In particular, from the definition of $W_{k}$ one knows that $\omega$ is not identically zero on $J_{k}(f)$.

In the proof of (1.2) they applied this lemma, taking $\delta=1$ and $k \geqq n$ fixed, and increasing $q \rightarrow \infty$.

Since $A \times W_{k}$ is the Zariski closure of the transcendental holomorphic mapping $J_{k}(f)$ : $\mathbf{C} \rightarrow J_{k}(A) \cong A \times \mathbf{C}^{n k}$, the variety $W_{k}$ must be allowed to be quite arbitrary. In fact, 
in the proof of the above Lemma 2, [SiY97], the fact that $A \times W_{k}$ was defined to be the Zariski closure of the $k$-jet lifting of $f: \mathbf{C} \rightarrow A$ with Zariski dense image was not used at all except for the last statement, and hence Lemma 2 (except for the last statement) should be true for arbitrary non-empty subvariety $W_{k} \subset \mathbf{C}^{n k}$ and ample $D$ on $A$, if the proof were correct. This is a very different point from our proof (cf. the proof of Lemma 5.4). But, we then deduce some contradictory conclusions as follows.

(a) We take an ample divisor $D$ on $A$ such that it contains a translate of a non-trivial Abelian subvariety $A^{\prime}$ (cf. $\left[\mathrm{NO} \frac{84}{90}\right]$, Example (6.4.13) for such an example). Let $g: \mathbf{C} \rightarrow A^{\prime}$ be a one-parameter subgroup with Zariski dense image. We regard $g$ as a holomorphic curve into $A$, and set $f(z)=g(z)+a$ with $a \in A \backslash D$. Then $f$ is a holomorphic curve such that $f(\mathbf{C}) \not \subset D$, and $W_{k}$ consists of only one point for every $k \geqq 1$. We obtain $A \times W_{k} \cong A$. Through this isomorphism, we have that

$$
A^{\prime} \subset J_{k}(D) \cap\left(A \times W_{k}\right) \varsubsetneqq A .
$$

Let $\mathcal{I}_{k}=\mathcal{I}\left(J_{k}(D) \cap\left(A \times W_{k}\right)\right)$ denote the ideal sheaf of $J_{k}(D) \cap\left(A \times W_{k}\right)(\subset A)$. Note that any jet differential of any weight $m$ restricted to $A \times W_{k}$ is reduced to a jet differential of weight 0 , for $W_{k}$ consists of one point. Then Lemma 2 should imply that for all $q \geqq 1$

$$
H^{0}\left(A, \mathcal{O}\left((L(D))^{\delta}\right) \otimes \mathcal{I}_{k}^{q}\right) \neq\{0\}
$$

where $\mathcal{O}\left((L(D))^{\delta}\right)$ denotes the sheaf of germs of holomorphic sections of $(L(D))^{\delta}$. Since $A^{\prime} \subset J_{k}(D) \cap\left(A \times W_{k}\right)$, the ideal sheaf $\mathcal{I}=\mathcal{I}\left(A^{\prime}\right)$ of $A^{\prime}$ contains $\mathcal{I}_{k}$. Therefore,

$$
H^{0}\left(A, \mathcal{O}\left((L(D))^{\delta}\right) \otimes \mathcal{I}^{q}\right) \neq\{0\}, \quad \forall q \geqq 1,
$$

and hence the infinite dimensionality of $H^{0}\left(A, \mathcal{O}\left((L(D))^{\delta}\right)\right)$ would follow, where $\delta$ had been fixed. This is clearly absurd. This observation implies that the Zariski denseness of the image $f(\mathbf{C})$ in $A$ must be used essentially.

(b) We also observe that Lemma 2 is not valid even for $f: \mathbf{C} \rightarrow A$ with Zariski dense image, and moreover that $k$ cannot be fixed as stated in Lemma 2 . Let $k \geqq n$ be any fixed. Let $f: \mathbf{C} \rightarrow A$ be a one-parameter subgroup with Zariski dense image. Let $D$ be an ample divisor on $A$ containing the zero $0 \in A$ such that $f(\mathbf{C})$ is tangent highly enough to $D$ at 0 so that $J_{k} f(0) \in J_{k}(D)$, but $f(\mathbf{C}) \not \subset D$. Let $\mathfrak{m}_{0}$ be the maximal ideal sheaf of the structure sheaf $\mathcal{O}_{A}$ at 0 . Since $W_{k}$ consists of only one point, $A \times W_{k} \cong A$, and through this isomorphism $0 \in J_{k}(D) \cap\left(A \times W_{k}\right)$. Therefore we have that $\mathcal{I}_{k}=$ $\mathcal{I}\left(J_{k}(D) \cap\left(A \times W_{k}\right)\right) \subset \mathfrak{m}_{0}$. As in (a), Lemma 2 should imply that

$$
H^{0}\left(A, \mathcal{O}\left((L(D))^{\delta}\right) \otimes \mathfrak{m}_{0}^{q}\right) \supset H^{0}\left(A, \mathcal{O}\left((L(D))^{\delta}\right) \otimes \mathcal{I}_{k}^{q}\right) \neq\{0\}, \quad \forall q \geqq 1 .
$$

Thus we would obtain that $\operatorname{dim} H^{0}\left(A, \mathcal{O}\left((L(D))^{\delta}\right)\right)=\infty$; this is a contradiction. 
(c) The reason of the contradictions observed in (a) and (b) with respect to the above Lemma 2 comes from the use of the semi-continuity theorem for a non-flat family of coherent ideal sheaves. They used a deformation technique of the given ample divisor $D$. That is, taking a generic small deformation family $D(t), t \in \Delta(1)$ on $A$ with $D(0)=D$, they considered the family of ideals, $\left\{\left(\mathcal{I}\left(J_{k}(D(t)) \cap\left(A \times W_{k}\right)\right)\right)^{q}\right\}_{t \in \Delta(1)}$ for $k \geqq n$ and $q \geqq 1$. More precisely, they worked on the compactification $\overline{J_{k}(A)}=A \times \mathbf{P}^{n k}(\mathbf{C})$ of $J_{k}(A) \cong A \times \mathbf{C}^{n k}$. Let $\overline{J_{k}(D(t))}\left(\right.$ resp. $\left.\bar{W}_{k}\right)$ denote the closure of $J_{k}(D(t))$ (resp. $\left.W_{k}\right)$ in $\overline{J_{k}(A)}$ (resp. $\mathbf{P}^{n k}(\mathbf{C})$ ). To apply the semi-continuity theorem of the dimension of cohomology groups, one needs the flatness of the family, $\left\{\mathcal{I}\left(\overline{J_{k}(D(t))} \cap\left(A \times \bar{W}_{k}\right)\right)\right\}_{t \in \Delta(1)}$. In general, the constructed family $\left\{\mathcal{I}\left(\overline{J_{k}(D(t))} \cap\left(A \times \bar{W}_{k}\right)\right)\right\}_{t \in \Delta(1)}$ may not be flat, since there may be a "jump" of the supports of those ideals. This fact tells us the difficulty to apply the deformation technique to obtain the second main theorem in general. Because of its own interest we give such an example in what follows.

Let $E$ be an elliptic curve defined by the square lattice, $\mathbf{Z}+i \mathbf{Z}$, and set $A=E \times E$. Let $(x, y)$ be a local flat coordinate system of $A$, and define a holomorphic curve $f: \mathbf{C} \rightarrow A$ by

$$
f: z \in \mathbf{C} \rightarrow(z, \alpha z) \in A,
$$

where $\alpha$ is an irrational number. Then the image $f(\mathbf{C})$ is Zariski dense in $A$. The 2-jet lifting of $f$ is given by

$$
J_{2}(f)(z)=((z, \alpha z),(1, \alpha),(0,0)) \in J_{2}(A) \cong A \times \mathbf{C}^{2} \times \mathbf{C}^{2} .
$$

Thus,

$$
W_{2}=((1, \alpha),(0,0)) \text {. }
$$

Let $L$ be a sufficiently ample line bundle over $A$ such that $L$ carries a global holomorphic section $\sigma(x, y)$ whose germ at $(0,0)$ is written as

$$
-y^{2}+x^{3}+\alpha^{2} x^{2}+x^{4} G(x, y) .
$$

Let $D$ be the divisor defined by the zero locus of $\sigma$. By an easy computation one sees

$$
J_{2}(D)_{(0,0)}=\{(0,0)\} \times \mathbf{C}(1, \pm \alpha) \times \mathbf{C}^{2} .
$$

Therefore we have

$$
J_{2}(D) \cap\left(A \times W_{2}\right) \ni((0,0),(1, \alpha),(0,0)) .
$$

For small $t \in \mathbf{C}$ we consider a generic deformation $D(t)$ defined by

$$
-y^{2}+x^{3}+\alpha^{2} x^{2}+x^{4} G(x, y)+t H(x, y)=0 .
$$


We look for a point $\left(x_{0}, y_{0}\right) \in D(t)$ near $(0,0)$ with $t \neq 0$ such that $\left(x_{0}, y_{0}\right) \times(1, \alpha) \times(0,0) \in$ $J_{2}(D(t)) \cap\left(A \times W_{2}\right)$. First we have

$$
-y_{0}^{2}+x_{0}^{3}+\alpha^{2} x_{2}^{2}+x_{0}^{4} G\left(x_{0}, y_{0}\right)+t H\left(x_{0}, y_{0}\right)=0 .
$$

Set

$$
\begin{aligned}
\phi(z)= & -\left(y_{0}+\alpha z\right)^{2}+\left(x_{0}+z\right)^{3}+\alpha^{2}\left(x_{0}+z\right)^{2}+\left(x_{0}+z\right)^{4} G\left(x_{0}+z, y_{0}+\alpha z\right) \\
& +t H\left(x_{0}+z, y_{0}+\alpha z\right) .
\end{aligned}
$$

Then one gets

$$
\begin{aligned}
\phi^{\prime}(z)= & -2 \alpha\left(y_{0}+\alpha z\right)+3\left(x_{0}+z\right)^{2}+2 \alpha^{2}\left(x_{0}+z\right)+\left(x_{0}+z\right)^{3} G_{1}\left(x_{0}+z, y_{0}+\alpha z\right) \\
& +t H_{x}\left(x_{0}+z, y_{0}+\alpha z\right)+t \alpha H_{y}\left(x_{0}+z, y_{0}+\alpha z\right),
\end{aligned}
$$

where $G_{1}$ is a naturally defined holomorphic function. Hence,

$$
\begin{aligned}
\phi^{\prime}(0)= & -2 \alpha y_{0}+3 x_{0}^{2}+2 \alpha^{2} x_{0}+x_{0}^{3} G_{1}\left(x_{0}, y_{0}\right) \\
& +t H_{x}\left(x_{0}, y_{0}\right)+t \alpha H_{y}\left(x_{0}, y_{0}\right)=0 .
\end{aligned}
$$

Taking the second derivative, we have

$$
\begin{aligned}
\phi^{\prime \prime}(z)= & 6\left(x_{0}+z\right)+\left(x_{0}+z\right)^{2} G_{2}\left(x_{0}+z, y_{0}+\alpha z\right) \\
& +t H_{x x}\left(x_{0}+z, y_{0}+\alpha z\right)+2 t \alpha H_{x y}\left(x_{0}+z, y_{0}+\alpha z\right)+t \alpha^{2} H_{y y}\left(x_{0}+z, y_{0}+\alpha z\right),
\end{aligned}
$$

and so

$$
\begin{aligned}
\phi^{\prime \prime}(0)= & \left(6+x_{0} G_{2}\left(x_{0}, y_{0}\right)\right) x_{0} \\
& +\left(H_{x x}\left(x_{0}, y_{0}\right)+2 \alpha H_{x y}\left(x_{0}, y_{0}\right)+\alpha H_{y y}\left(x_{0}, y_{0}\right)\right) t=0 .
\end{aligned}
$$

We may assume that for $\left(x_{0}, y_{0}\right)$ close to $(0,0)$

$$
6+x_{0} G_{2}\left(x_{0}, y_{0}\right) \neq 0
$$

Thus we may write

$$
x_{0}=t \psi\left(t, y_{0}\right) \text {. }
$$

We substitute this to (5.32), and get

$$
\begin{aligned}
& -2 \alpha y_{0}+3 t^{2} \psi^{2}\left(t, y_{0}\right)+2 \alpha^{2} t \psi\left(t, y_{0}\right)+t^{3} \psi^{3}\left(t, y_{0}\right) G_{1}\left(t \psi\left(t, y_{0}\right), y_{0}\right) \\
& +t H_{x}\left(t \psi\left(t, y_{0}\right), y_{0}\right)+t \alpha H_{y}\left(t \psi\left(t, y_{0}\right), y_{0}\right)=0
\end{aligned}
$$


Therefore we have

$$
y_{0}=t \lambda(t), \quad x_{0}=t \psi(t, t \lambda(t))=t \mu(t) .
$$

Then we substitute these to (5.31), and obtain

$$
-t^{2} \lambda^{2}(t)+t^{3} \mu^{3}(t)+\alpha^{2} t^{2} \mu^{2}(t)+t^{4} \mu^{4}(t) G(t \mu(t), t \lambda(t))+t H(t \mu(t), t \lambda(t))=0 .
$$

Since $t \neq 0$, we have

$$
-t \lambda^{2}(t)+t^{2} \mu^{3}(t)+\alpha^{2} t \mu^{2}(t)+t^{3} \mu^{4}(t) G(t \mu(t), t \lambda(t))+H(t \mu(t), t \lambda(t))=0 .
$$

We assume a generic condition, $H(0,0) \neq 0$; equation (5.34) is not trivial. Thus, $t$ satisfying (5.34) is isolated, and cannot approach 0 .

It follows that there is a neighborhood $U \subset J_{2}(A)$ of $((0,0),(1, \alpha),(0,0)) \in J_{2}(D(0)) \cap$ $\left(A \times W_{2}\right)$ such that for every small $t \neq 0$,

$$
J_{2}(D(t)) \cap\left(A \times W_{2}\right) \cap U=\emptyset .
$$

Therefore the ideal family $\left\{\mathcal{I}\left(\overline{J_{k}(D(t))} \cap\left(A \times \bar{W}_{k}\right)\right)\right\}_{t \in \Delta(1)}$ is not flat.

Remark 5.35 It is an interesting problem to see if the truncation level $k_{0}$ of the counting function $N_{k_{0}}\left(r ; f^{*} D\right)$ in the Main Theorem can be taken as a function only in $\operatorname{dim} A$. By the above proof, it would be sufficient to find a natural number $k$ such that $\pi_{2}\left(J_{k}(\bar{D} ; \log \bar{A} \cap\right.$ $\partial A)) \cap W_{k} \neq W_{k}$. Note that $\operatorname{dim} \pi_{2}\left(J_{k}(\bar{D} ; \log \bar{A})\right) \leqq \operatorname{dim} J_{k}(\bar{D} ; \log \bar{A})=(n-1)(k+1)$. Thus, if $\operatorname{dim} W_{k}>(n-1)(k+1)$ we may set $k_{0}=k$. For example, if $J_{n}(f)(\mathbf{C})$ is Zariski dense in $J_{n}(A)$, then $\operatorname{dim} W_{n}=n^{2}$. Since $\operatorname{dim} \pi_{2}\left(J_{n}(\bar{D} ; \log \partial A)\right)=n^{2}-1$, we may set $k_{0}=n$.

\section{Applications.}

Let the notation be as in the previous section. Here we assume that $A$ is an Abelian variety and $D$ is reduced and hyperbolic; in this special case, $D$ is hyperbolic if and only if $D$ contains no translate of a one-parameter subgroup of $A$. Cf. [NO $\left.\frac{84}{90}\right]$, [La87] and [Ko98] for the theory of hyperbolic complex spaces.

Theorem 6.1 Let $D \subset A$ be hyperbolic and $d_{0}$ be the highest order of tangency of $D$ with translates of one-parameter subgroups. Let $\pi: X \rightarrow A$ be a finite covering space such that its ramification locus contains $D$ and the ramification order over $D$ is greater than $d_{0}+1$. Then $X$ is hyperbolic. 
Proof. By Brody's theorem (cf., e.g., $\left[\mathrm{NO} \frac{84}{90}\right]$, Theorem (1.7.3)) it suffices to show that there is no non-constant holomorphic curve $g: \mathbf{C} \rightarrow X$ such that the length $\left\|g^{\prime}(z)\right\|$ of the derivative $g^{\prime}(z)$ of $g(z)$ with respect to an arbitrarily fixed Finsler metric on $X$ is bounded. Set $f(z)=\pi(g(z))$. Then the length $\left\|f^{\prime}(z)\right\|$ with respect to the flat metric is bounded, too, and hence $f^{\prime}(z)$ is constant. Thus, $f(z)$ is a translate of a one-parameter subgroup. By definition we may take $k_{0}=d_{0}+1$ in (5.7). Take $d\left(>d_{0}+1\right)$ so that $X$ ramifies over $D$ with order at least $d$. Then we have that $N_{1}\left(r ; f^{*} D\right) \leqq \frac{1}{d} N\left(r ; f^{*} D\right)$. Hence it follows from the Main Theorem that

$$
\begin{aligned}
T_{f}(r ; L(D)) & =N_{d_{0}+1}\left(r, f^{*} D\right)+O(\log r) \leqq\left(d_{0}+1\right) N_{1}\left(r ; f^{*} D\right)+O(\log r) \\
& \leqq \frac{d_{0}+1}{d} N\left(r ; f^{*} D\right)+O(\log r) \leqq \frac{d_{0}+1}{d} T_{f}(r ; L(D))+O(\log r) .
\end{aligned}
$$

Since $T_{f}(r ; L(D)) \geqq c_{0} r^{2}$ with a constant $c_{0}>0, d \leqq d_{0}+1 ;$ this is a contradiction. Q.E.D.

Remark. In the special case of $\operatorname{dim} X=\operatorname{dim} A=2$, C.G. Grant [Gr86] proved that if $X$ is of general type and $X \rightarrow A$ is a finite (ramified) covering space, then $X$ is hyperbolic. When $\operatorname{dim} X=\operatorname{dim} A=2, D$ is an algebraic curve, and hence the situation is much simpler than the higher dimensional case.

Theorem 6.2 Let $f: \mathbf{C} \rightarrow A$ be a 1-parameter analytic subgroup in $A$ with $a=f^{\prime}(0)$. Let $D$ be an effective divisor on $A$ with the Riemann form $H(\cdot, \cdot)$. Then we have

$$
N\left(r ; f^{*} D\right)=H(a, a) \pi r^{2}+O(\log r) .
$$

Proof. Note that the first Chern class $c_{1}(L(D))$ is represented by $i \partial \bar{\partial} H(w, w)$. It follows from (2.1) and Lemma 5.1 that

$$
\begin{aligned}
N\left(r ; f^{*} D\right) & =T_{f}(r ; L(D))+O(\log r) \\
& =\int_{0}^{r} \frac{d t}{t} \int_{\Delta(t)} i H(a, a) d z \wedge d \bar{z}+O(\log r) \\
& =H(a, a) \pi r^{2}+O(\log r) .
\end{aligned}
$$

Q.E.D.

Remark 6.3 In the case where $f(\mathbf{C})$ is Zariski dense in $A, A x([A x 72])$ proved the following estimate,

$$
0<\varliminf_{r \rightarrow \infty} \frac{n\left(r, f^{*} D\right)}{r^{2}} \leqq \varlimsup_{r \rightarrow \infty} \frac{n\left(r, f^{*} D\right)}{r^{2}}<\infty
$$

which is equivalent to

$$
0<\varliminf_{r \rightarrow \infty} \frac{N\left(r, f^{*} D\right)}{r^{2}} \leqq \varlimsup_{r \rightarrow \infty} \frac{N\left(r, f^{*} D\right)}{r^{2}}<\infty .
$$




\section{References}

[Ah41] L. V. Ahlfors, The theory of meromorphic curves, Acta Soc. Sci. Fennicae, Nova Ser. A. 3 (1941), 3-31.

[AN91] Y. Aihara and J. Noguchi, Value distribution of meromorphic mappings into compactified locally symmetric spaces, Kodai Math. J. 14 (1991), 320-334.

[Ax72] J. Ax, Some topics in differential algebraic geometry II, Amer. J. Math. 94 (1972), 1205-1213.

[Bl26] Bloch, A.: Sur les systèmes de fonctions uniformes satisfaisant à l'équation d'une variété algébrique dont l'irrégularité dépasse la dimension. J. Math. Pures Appl. 5 (1926), 19-66.

[CG72] J. Carlson and P. Griffiths, A defect relation for equidimensional holomorphic mappings between algebraic varieties, Ann. Math. 95 (1972), 557-584.

[Ca33] H. Cartan, Sur les zéros des combinaisons linéaires de $p$ fonctions holomorphes données, Mathematica 7 (1933), 5-31.

[DL97] G. Dethloff and S.S.Y. Lu, Logarithmic projective jet bundles and applications, preprint, 1997.

[ES92] A.E. Eremenko and M.L. Sodin, The value distribution of meromorphic functions and meromorphic curves from the view point of potential theory, St. Petersburg Math. J. 3 (1992) No. 1, 109-136.

[Gr86] C.G. Grant, Entire holomorphic curves in surfaces, Duke Math. J. 53 (1986), 345358.

[GK73] P. Griffiths and J. King, Nevanlinna theory and holomorphic mappings between algebraic varieties, Acta Math. 130 (1973), 145-220.

[Ha64] W.K. Hayman, Meromorphic Functions, Oxford Math. Monographs, Oxford University Press, London, 1964.

[Kr98] R. Kobayashi, Holomorphic curves in Abelian varieties: The second main theorem and applications, preprint, 1998.

[Ko98] S. Kobayashi, Hyperbolic Complex Spaces, Grundlehren der mathematischen Wissenschaften 318, Springer-Verlag, Berlin-Heidelberg, 1998.

[La87] S. Lang, Introduction to Complex Hyperbolic Spaces, Springer-Verlag, New YorkBerlin-Heidelberg, 1987.

[Mc96] M. McQuillan, A dynamical counterpart to Faltings' "Diophantine approximation on Abelian varieties", I.H.E.S. preprint, 1996.

[No77] J. Noguchi, Holomorphic curves in algebraic varieties, Hiroshima Math. J. 7 (1977), 833-853. 
[No81] J. Noguchi, Lemma on logarithmic derivatives and holomorphic curves in algebraic varieties, Nagoya Math. J. 83 (1981), 213-233.

[No86] J. Noguchi, Logarithmic jet spaces and extensions of de Franchis' theorem, Contributions to Several Complex Variables, pp. 227-249, Aspects Math. No. 9, Vieweg, Braunschweig, 1986.

[No96] J. Noguchi, On Nevanlinna's second main theorem, Geometric Complex Analysis, Proc. the Third International Research Institute, Math. Soc. Japan, Hayama, 1995, pp. 489-503, World Scientific, Singapore, 1996.

[No98] J. Noguchi, On holomorphic curves in semi-Abelian varieties, Math. Z. 228 (1998), 713-721.

[NW99] J. Noguchi and J. Winkelmann, Holomorphic Curves and Integral Points off Divisors, preprint, 1999.

$\left[\mathrm{NO} \frac{84}{90}\right]$ J. Noguchi and T. Ochiai, Geometric Function Theory in Several Complex Variables, Japanese edition, Iwanami, Tokyo, 1984; English Translation, Transl. Math. Mono. 80, Amer. Math. Soc., Providence, Rhode Island, 1990.

[Si87] Y.-T. Siu, Defect relations for holomorphic maps between spaces of different dimensions, Duke Math. J. 55 (1987), 213-251.

[SiY96] Y.-T. Siu and S.-K. Yeung, A generalized Bloch's theorem and the hyperbolicity of the complement of an ample divisor in an Abelian variety, Math. Ann. 306 (1996), 743-758.

[SiY97] Y.-T. Siu and S.-K. Yeung, Defects for ample divisors of Abelian varieties, Schwarz lemma, and hyperbolic hypersurfaces of low degrees, Amer. J. Math. 119 (1997), 1139-1172.

[St53/54] W. Stoll, Die beiden Hauptsätze der Wertverteilungstheorie bei Funktionen mehrerer komplexer Veränderlichen (I); (II), Acta Math. 90 (1953), 1-115; ibid, Acta Math. 92 (1954), 55-169.

[We58] Weil, A.: Introduction à l'étude des variétés kähleriennes, Hermann, Paris, 1958.

Graduate School of Mathematical Sciences University of Tokyo Komaba, Meguro,Tokyo 153-8914 e-mail: noguchi@ms.u-tokyo.ac.jp

Mathematisches Institut Rheinsprung 21 CH-4053 Basel Switzerland e-mail: jwinkel@member.ams.org URL: http://www.cplx.ruhr-uni-bochum.de/ jw/index-e.html 
Research Institute for Mathematical Sciences Kyoto University Oiwake-cho, Sakyoku, Kyoto 606-8502 e-mail: ya@kurims.kyoto-u.ac.jp 